\title{
LTR-retrotransposon dynamics in common fig (Ficus carica L.) genome
}

\author{
Alberto Vangelisti ${ }^{\dagger}$, Samuel Simoni ${ }^{\dagger}$, Gabriele Usai, Maria Ventimiglia, Lucia Natali, Andrea Cavallini ${ }^{*}$, \\ Flavia Mascagni ${ }^{*}$ and Tommaso Giordani
}

\begin{abstract}
Background: Long Terminal Repeat retrotransposons (LTR-REs) are repetitive DNA sequences that constitute a large part of the genome. The improvement of sequencing technologies and sequence assembling strategies has achieved genome sequences with much greater reliability than those of the past, especially in relation to repetitive DNA sequences.

Results: In this study, we analysed the genome of Ficus carica L., obtained using third generation sequencing technologies and recently released, to characterise the complete complement of full-length LTR-REs to study their dynamics during fig genome evolution. A total of 1867 full-length elements were identified. Those belonging to the Gypsy superfamily were the most abundant; among these, the Chromovirus/Tekay lineage was the most represented. For the Copia superfamily, Ale was the most abundant lineage. Measuring the estimated insertion time of each element showed that, on average, Ivana and Chromovirus/Tekay were the youngest lineages of Copia and Gypsy superfamilies, respectively. Most elements were inactive in transcription, both constitutively and in leaves of plants exposed to an abiotic stress, except for some elements, mostly belonging to the Copia/Ale lineage. A relationship between the inactivity of an element and inactivity of genes lying in close proximity to it was established.
\end{abstract}

Conclusions: The data reported in this study provide one of the first sets of information on the genomic dynamics related to LTR-REs in a plant species with highly reliable genome sequence. Fig LTR-REs are highly heterogeneous in abundance and estimated insertion time, and only a few elements are transcriptionally active. In general, the data suggested a direct relationship between estimated insertion time and abundance of an element and an inverse relationship between insertion time (or abundance) and transcription, at least for Copia LTR-REs.

Keywords: Genome structure and evolution, Long terminal repeat retrotransposons, Plant retrotransposon dynamics, Retrotransposon expression, Retrotransposon insertion time, Ficus carica L

\section{Background}

The fig tree (Ficus carica L.) belongs to the Moraceae family and is a deciduous fruit tree considered amongst the oldest domesticated tree species [1]. During the last decades the interest in fig considerably increased because of both the nutraceutical properties of the fruit

\footnotetext{
*Correspondence: andrea.cavallini@unipi.it; flavia.mascagni@unipi.it ${ }^{+}$Alberto Vangelisti and Samuel Simoni contributed equally to this work. Dipartimento di Scienze Agrarie, Alimentari e Agro-ambientali, Università di Pisa, Via del Borghetto 80, 56124 Pisa, Italy
}

and economic value of this species [2,3]. F. carica cultivation is spread throughout the Mediterranean area, Middle East, and Asia. These regions are affected by a high temperate climate during the summer, which increases salinity in the soil due to reduced water availability [4]. The fig tree has showed moderate resistance to saline stress compared to other fruit trees $[5,6]$. Recently, the $F$. carica genome has been completely sequenced [1], showing a total of $\sim 333 \mathrm{Mbp}$ arranged in 13 pseudo-chromosomes, containing 37,840 genes,

(c) The Author(s). 2021 Open Access This article is licensed under a Creative Commons Attribution 4.0 International License, which permits use, sharing, adaptation, distribution and reproduction in any medium or format, as long as you give appropriate credit to the original author(s) and the source, provide a link to the Creative Commons licence, and indicate if changes were made. The images or other third party material in this article are included in the article's Creative Commons licence, unless indicated otherwise in a credit line to the material. If material is not included in the article's Creative Commons licence and your intended use is not permitted by statutory regulation or exceeds the permitted use, you will need to obtain permission directly from the copyright holder. To view a copy of this licence, visit http://creativecommons.org/licenses/by/4.0/ The Creative Commons Public Domain Dedication waiver (http://creativecommons.org/publicdomain/zero/1.0/) applies to the data made available in this article, unless otherwise stated in a credit line to the data. 
mostly composed of repeated sequences of which long terminal repeat retrotransposons (LTR-REs) constitute the vast majority [1]. However, a comprehensive analysis of fig LTR-REs is still lacking.

Retrotransposons (REs) are usually widespread in all eukaryotes and constitute a large part of the genome, especially in plant species [7]. They can move across chromosomes, thus, multiplying their number into the genome of host plants. In fact, REs replicate by a 'copy and paste' mechanism, exploiting the expression of an RNA intermediate that is converted into cDNA before being inserted back into a different chromosomal location. The most abundant order of REs in plant species shows two long terminal repeats (LTRs) flanking a polyprotein region, containing encoding domains necessary to replicate the sequence, such as protease, reverse transcriptase, RNAse $\mathrm{H}$, and an integrase, with an additional GAG domain that encodes for virus-like particles protein $[7,8]$. The majority of plant LTR-REs are represented by the two main superfamilies, Copia and Gypsy, which differ principally in sequence divergence and the organization of polyprotein domains. Based on sequence similarities, these elements can be distinguished into several lineages $[7,9,10]$.

The RE component of a plant genome is subject to turnover [11, 12]; in fact, REs may increase their number in a relatively short time. However, REs may also be removed from the genome through unequal homologous and illegitimate recombination $[13,14]$.

Retrotransposons play an important role in the evolution of a species because their mobility produces genetic variation [15]. They can favour chromosome rearrangements through illegitimate recombination [13] between sites lying far in the same chromosome or in different chromosomes [16]. Moreover, REs may insert within or near a gene, altering its coding sequence or its splicing pattern [17]. Perhaps more importantly, the insertion or removal of a RE in proximity of a gene may change its transcription regulation, altering its expression rate in response to different stimuli [18-21].

Because of the potentially harmful effects of their mobility, REs are generally epigenetically inactivated by the host, for example, through DNA methylation [22, 23]. Consequently, the insertion of a RE in a locus may lead to modification of the epigenetic setting of that insertion site [17]. As a matter of fact, REs are known to regulate the epigenetic setting of the genome and chromatin organisation and structure [17].

Nevertheless, LTR-REs can elude silencing machinery and move into new sites, leading to potential genetic changes. For example, the activation of LTR-REs has been reported in many plant species; in many cases, the regulation of RE expression was shown to depend on external stimuli, such as those related to biotic and abiotic stresses [24-27].

In genome sequences obtained using high throughput second-generation sequencing technologies which use relatively short DNA sequences to be assembled, the assembly of LTR-REs can be complicated by the fact that such REs are repeated (in many cases, highly repeated) in the genome and are by far longer than a single read. Furthermore, in some cases, the assembled sequence may be composed of sequence reads derived from multiple copies of the repetitive element collapsed together, resulting in a consensus rather than in a real sequence.

Third-generation sequencing (TGS) technologies [28], that produce very long sequence reads, have allowed the production of high-quality genome assemblies [29-31], overcoming many of the problems related to short-read sequencing assemblies, such as the resolution of repetitive sequences $[22,32]$.

Recently, a high-quality genome sequence of Ficus carica cv. Dottato [1] was produced using single-molecule, real-time (SMRT) sequencing developed by Pacific Biosciences (PacBio, Menlo Park, CA, USA), the first attempt to sequence the genome of this heterozygous species by long-read assembly, thus providing a highquality genomic resource. In fact, PacBio technology can successfully resolve the complex repetitive fraction of most genomes, such as those of plants, by spanning complete repetitive regions with unique sequences. The availability of such a contiguous reference genome assembly is a crucial prerequisite for the correct identification of repeats by a de novo approach.

In the previous work [1], repeated sequences were identified and subjected to a preliminary characterization. The repeated sequences were used to mask the assembly, thus obtaining the count of the global amount of bases associated to putative repeated regions, but no analysis were performed on single full-length elements. Here, we present a comprehensive study of fig LTR-REs, with the identification and complete characterisation of all full-length elements. In particular, after identification and structural characterisation, we analysed the abundance and estimated insertion time of each isolated element in the genome of $F$. carica. Furthermore, we used a transcriptomic approach to analyse the expression of LTR-REs during abiotic stress, i.e., in fig leaves from plants of the same cultivar (Dottato) exposed to short and prolonged periods of saline stress [33]. In fact, LTR-RE expression represents the first step of retrotransposon activation, with possible consequences on the genetic structure of a species. Finally, the data on abundance, age, and transcription activity of all LTR-REs were related to provide a global view on the dynamics of REs in a precise plant system. 


\section{Results}

Full-length LTR-REs isolation and structural characterisation

A structural analysis allowed us to identify a total of 1867 unique full-length long terminal repeat retrotransposons (LTR-REs).

A comparison between the two available fig genome assembly, the one produced using third generation sequencing technology [1] and the previous NGS-based assembly [34] showed that the number of retrieved full-length elements was by far higher using long-read sequencing. In fact, only 20 full-length LTR-REs versus 1867 could be retrieved from the NGS-based assembly. The total amount of 1887 LTR-REs were clustered using 90\% sequence similarity thresholds. Only sixteen out of 1867 LTR-REs clustered with elements of NGS-based assembly, further showing the large improvement in repeat assembly obtained using third generation sequencing technology.

Overall, 1010 out of 1867 LTR-REs showed all functional domains, whereas the remaining 857 elements lacked one or more functional domains, hence they can be considered as transpositionally non-autonomous.

We were able to annotate 1163 Gypsy elements (62.52\%) and 623 Copia elements (33.49\%), i.e., the number of Gypsy full-length elements was almost twofold higher than the Copia ones. A total of 74 full-length LTR-REs (3.97\%) remained unknown (Fig. 1).

With regards to the Copia superfamily, 319 Ale (51.20\%), 152 Angela (24.39\%), 4 Bianca (0.64\%), 34 Ikeros (5.45\%), 22 Ivana (3.53\%), 14 Sire (2.24\%), 41 TAR (6.58\%), and 37
Tork (5.93\%) elements were characterised (Fig. 1). The Ale lineage was by far predominant, followed by Angela. The other identified lineages were underrepresented.

As for the Gypsy superfamily, Chromovirus elements were the most abundant, being 835 out of 1163 (71.77\%), subdivided into 108 CRM (9.28\%), 15 Galadriel (1.28\%), 8 Reina (0.68\%), and 704 Tekay (60.53\%) elements. The other Gypsy lineages, Athila and Ogre-Tat were represented by 125 (10.74\%) and 203 (17.44\%) elements, respectively (Fig. 1).

Concerning the genomic abundance of LTRretrotransposons in the $F$. carica genome, mapping Illumina DNA reads to the full-length LTR-retrotransposons (see Methods) showed that Gypsy was the most plentiful superfamily. In particular, the most abundant lineage was Chromovirus/CRM, with an average coverage of 141.76, followed by Chromovirus/Tekay, Ogre-Tat/TatV, and Athila, showing average coverage of 26.23, 23.58, and 23.47, respectively (Fig. 2). The most represented lineages belonging to the Copia superfamily were $T A R$, with an average coverage of 29.06, followed by Ikeros (26.75 average coverage), Ivana (24.53), Angela (23.95), Ale (20.49), and Tork (19.59) (Fig. 2). Finally, LTR-retrotransposons that were not classified and named as 'unknown', showed an overall average coverage of 31.04 (Fig. 2).

\section{Insertion time of LTR-retrotransposons in the $F$. carica genome}

The insertion age of an LTR-RE can be estimated by counting nucleotide substitutions between the LTRs of

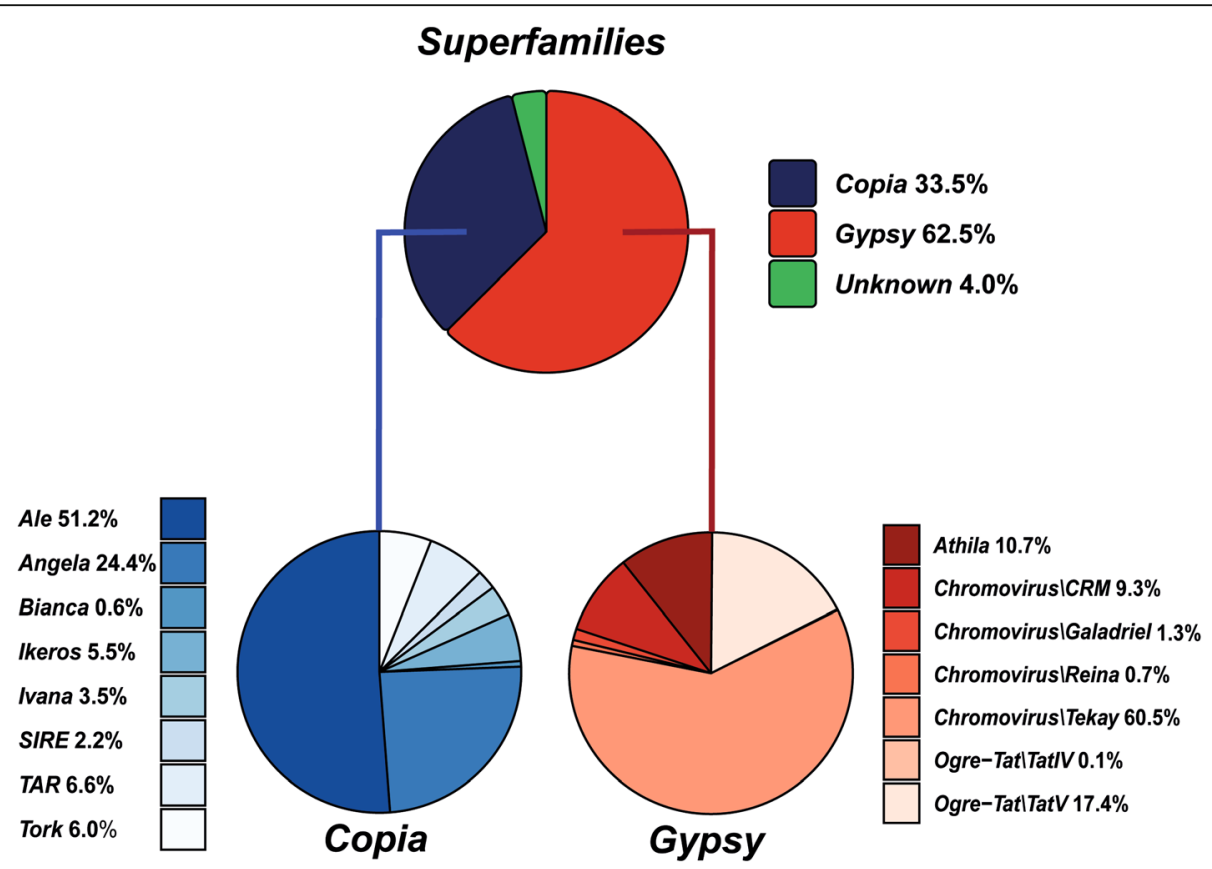

Fig. 1 Pie charts of the distribution of full-length LTR-REs in F. carica genome considering both superfamilies and Copia and Gypsy lineages 


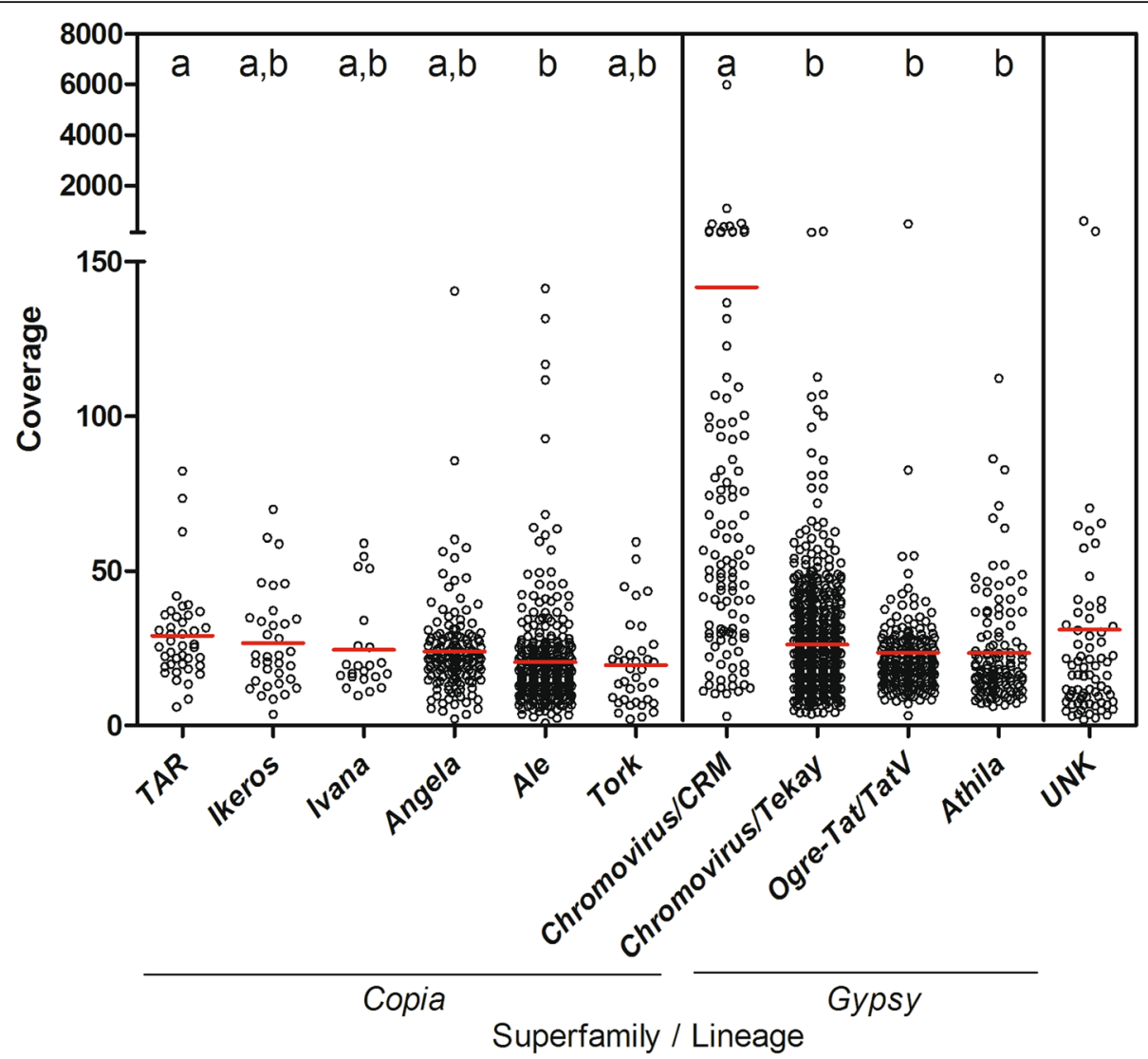

Fig. 2 Genomic abundance (showed as average coverage) of full-length LTR-REs for Copia and Gypsy lineages in the F. carica genome. Red bars represent the average value for each lineage. Significant differences (calculated by ANOVA) for each separate group of measurements are indicated by letters $a$ and b: groups sharing the same letter are not significantly different $(p<0.05)$ according to Tukey's test.

UNK = unknown superfamily

that same element, which should be identical immediately after the retrotransposition event and then accumulate mutations over time [35]. In our measurements, we applied the Populus trichocarpa synonymous substitution rate of $2.36 \times 10^{-9}$ (see Methods). Analyses were performed separately on the two LTR-RE superfamilies and on the most abundant Gypsy and Copia lineages.

The estimated insertion time of each fig full-length LTR-RE is reported in Fig. 3. On average, older elements belonged to Copia (7,9 MY on average) compared to Gypsy (6,1 MY). The oldest lineage belonging to the Copia superfamily was Ikeros (12,0 MY on average), while the youngest LTR-REs belonged to the Ivana (4,5 $\mathrm{MY})$ and Ale lineages $(6,4 \mathrm{MY})$. The oldest Gypsy lineage was Chromovirus/CRM (8,8 MY on average), whereas the youngest one was represented by Chromovirus/Tekay (5,3 MY). Unknown LTR-REs showed an average estimated insertion time of about 12,5 MY (Fig. 3).

\section{Expression analysis of LTR-retrotransposons in $\mathrm{F}$. carica}

The Illumina reads of cDNA from leaves of the control and salt stressed plants of $F$. carica at two time points,
24 and 48 days after the beginning of the experiment, were mapped on 1867 full-length LTR-REs. Overall, a total of 250,464 reads were aligned on the reference set of LTR-REs. On average, $0.22 \%$ of reads per library mapped on the sequence set, ranging from 0.16 to $0.31 \%$, depending on the library (Additional file 1 ).

Globally, low expression of full-length LTRs was detected for both Copia and Gypsy superfamilies with an average RPKM of 0.64 and 0.06 , respectively (Fig. 4). High percentages of unique reads (i.e., reads mapping to one LTR-RE only) were observed, spanning from 91.8 to $95.6 \%$. Such high percentages of unique reads should ensure high reliability to measured LTR-REs expression values.

Copia lineages showed slightly higher expression compared to Gypsy ones. The most expressed Copia lineage was Ale (mean RPKM =1.13), followed by Ivana (0.38) and Tork (0.23) (Fig. 4). The most expressed Gypsy lineages were Chromovirus/CRM (0.21), Athila (0.06), and Chromovirus/Tekay (0.04).

Considering unknown full-length LTR-REs, we retrieved an average RPKM of 1.04 (Fig. 4). In particular, 


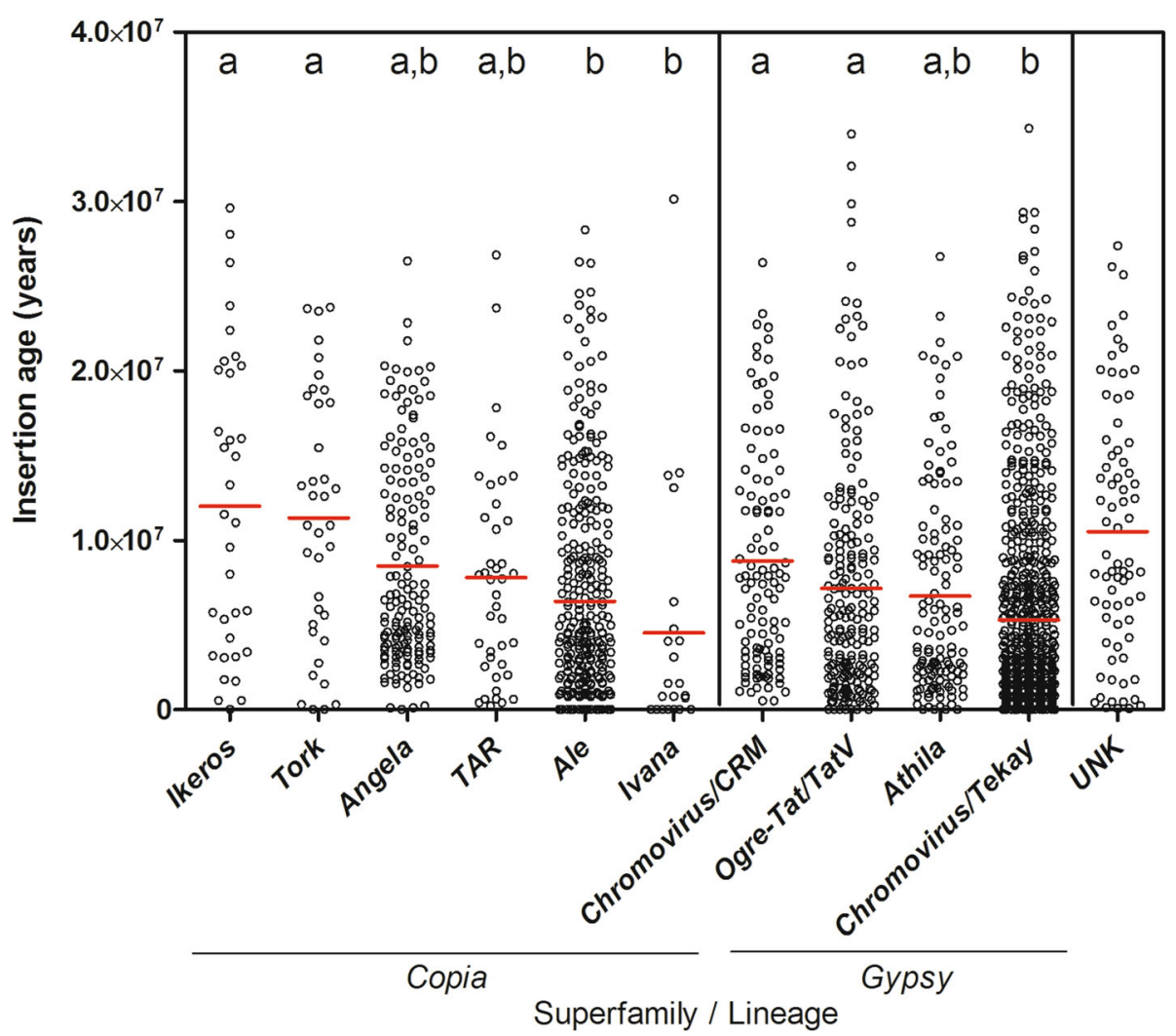

Fig. 3 Distribution of Copia and Gypsy full-length LTR-REs in the F. carica genome by insertion age. Red bars represent the average value for each lineage. Significant differences (calculated by ANOVA) for each separate group of measurements are indicated by letters a and b: groups sharing the same letter are not significantly different $(p<0.05)$ according to Tukey's test. UNK = unknown superfamily

one UNK element showed a very high RPKM. Further analysis on this element revealed the presence of two putative gene sequences within the unknown element, that might explain the high expression value of this LTR-RE.

We set RPKM $>1$, in at least one library, as a threshold to consider a full-length element as expressed during the experiments; based on this threshold, there were 153 expressed LTR-REs out of 1867. Considering the 153 expressed elements, 97 encoded all protein domains and therefore can be considered as intact and autonomous. Fifty-one expressed elements lacked one or more domains and 5 elements resulted to be devoid of domains.

The main fraction of expressed LTR-REs belonged to the Copia superfamily (81.0\%), followed by Gypsy (16.0\%) and unknown elements (3.0\%) (Fig. 5). Concerning lineages, the most represented Copia lineages were Ale (88.7\%) and Tork (4.0\%), whereas the most represented Gypsy lineage was Chromovirus (88.3\%), followed by Athila (8.3\%), as shown in Fig. 5. In particular, the expressed Chromovirus elements belonged to the four sublineages Tekay (60\%), Reina (20\%), CRM (15\%) and Galadriel (5\%).
LTR-retrotransposons transcription analysis in F. carica leaves of salt exposed plants

A total of 153 expressed LTR-REs (i.e., showing RPKM $>1$ in at least one RNA-seq library) during the saline stress in F. carica leaves were further investigated. Most of these REs (64) were expressed amongst all treatments, as shown in Fig. 6. Nevertheless, we found 22 LTR-REs uniquely expressed in the control and salt stressed leaves of $F$. carica after 24 days of saline treatment and one LTR-RE that was activated in control leaves after 48 days from the beginning of experiment (Fig. 6). We did not find any LTR-REs specifically expressed in the leaves of plants exposed to salt for 48 days.

Concerning the LTR-REs expression level we detected an average RPKM value of 2.74 amongst all libraries with a maximum of 89.56 , values similar to low/ medium-expressed reference genes that showed a stable expression both in control and salt stressed leaves (Additional File 2). Overall, we detected slightly higher expression during the first time point (24 days after beginning of experiment) for leaves of both control and stressed plants of $F$. carica (Additional File 3), although such differences proved to be not significant by ANOVA statistical analysis. 


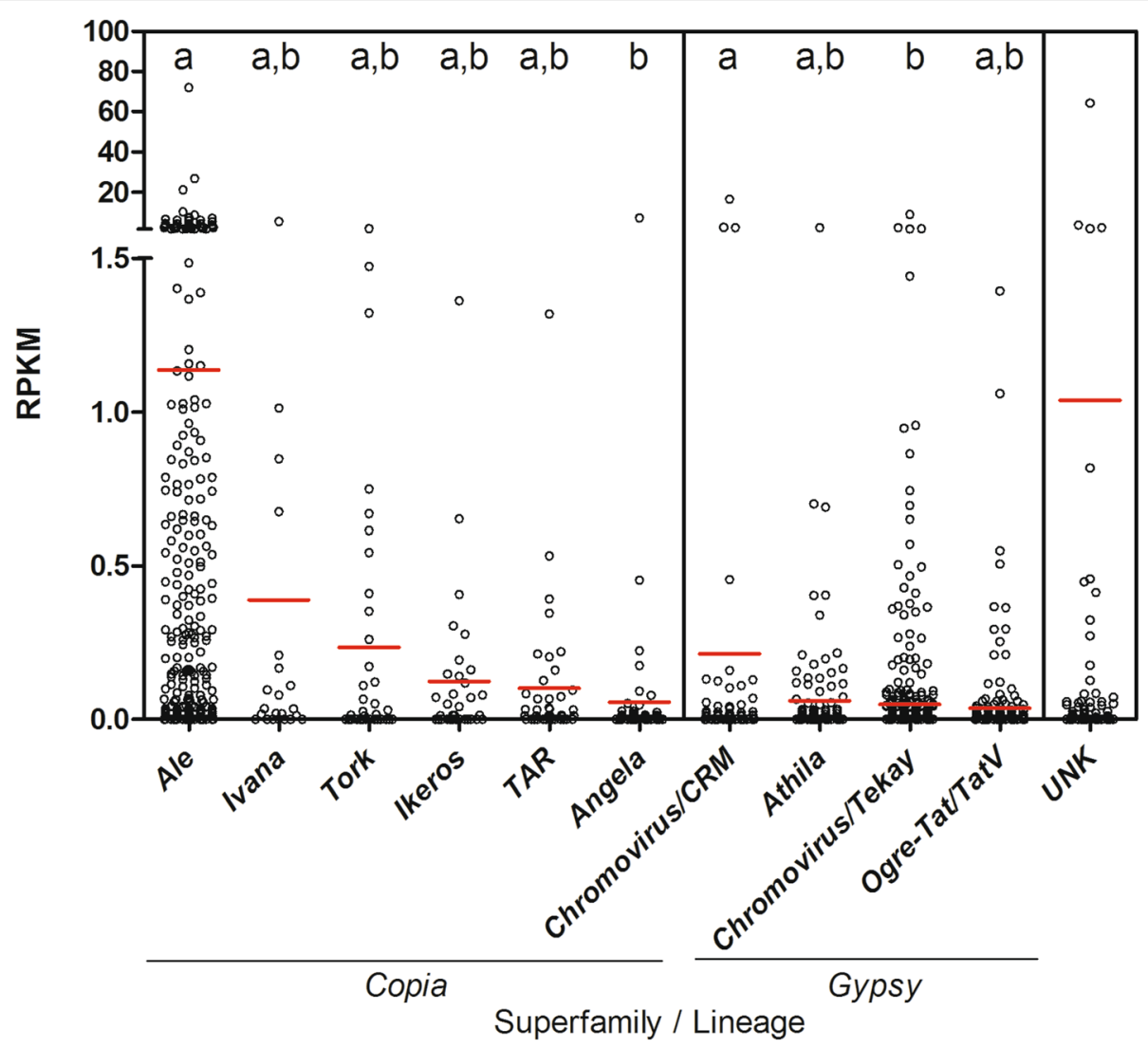

Fig. 4 Full-length LTR-RES RPKM distribution for each lineage in the F. carica genome. Red bars represent the average value for each lineage. Significant differences (calculated by ANOVA) for each separate group of measurements are indicated by letters a and b: groups sharing the same letter are not significantly different $(p<0.05)$ according to Tukey's test. UNK = unknown superfamily

To find differentially expressed LTR-REs during salt stress in fig leaves, we performed a pairwise test between the leaves of control and salt-treated plants at 24 and 48 days of treatment. We detected three Ale elements that were overexpressed after 24 days since the beginning of the experiment (Fig. 7a). At the second time point, we retrieved 13 activated LTR-REs (Fig. 7b), belonging to the Ale (10 elements), Chromovirus/Tekay (2), and Tork (1) lineages. Finally, one element whose superfamily was not identified was underregulated after 48 days of salt treatment (Fig. 7b).

\section{Expression of genes lying in close proximity to expressed full-length LTR-retrotransposons}

The expression and function of genes in the frame of 50 kbp up- and downstream to LTR-REs expressed (153 elements) or not expressed (1714) during salt stress in fig leaves were investigated. To analyse expression patterns, we separated genes in four categories: not expressed $($ RPKM $<$ $1)$, lowly expressed $(1<$ RPKM $<10)$, medium expressed $(10<$ RPKM < 100), and highly expressed (RPKM > 100). Overall we detected 1816 genes lying in close proximity to expressed LTR-REs. Of these, not expressed genes were the most abundant ( $48 \%$ average), followed by medium $(26 \%$ average), lowly ( $21 \%$ average), and highly expressed (5\% average) genes (Fig. 8). A comparison of expression level between genes lying in close proximity to expressed or not expressed LTR-REs showed a significantly higher percentage of highly and medium expressed genes lying close to active LTR-REs than to inactive ones in leaves of both the control and salt treated plants (Fig. 8).

In order to investigate the occurrence of specific GOterms in genes close to active LTR-REs, we used an enrichment analysis. This analysis compared the GO-terms in genes proximal to LTR-REs against the GO-terms of the whole $F$. carica transcriptome, by using a Fisher's exact Test. Only GO terms showing a FDR corrected $P$ Value $<0.05$ were considered. A total of 19 GO terms were specifically enriched compared to the whole $F$. carica transcriptome (Fig. 9). Among these, we found terms involved into lipid metabolic process, ribonucleotide binding, and steroid metabolic processes.

\section{LTR-retrotransposons dynamics in F. carica genome}

The relationships between expression, genome abundance, and estimated insertion time of full-length LTR- 


\section{Superfamilies}

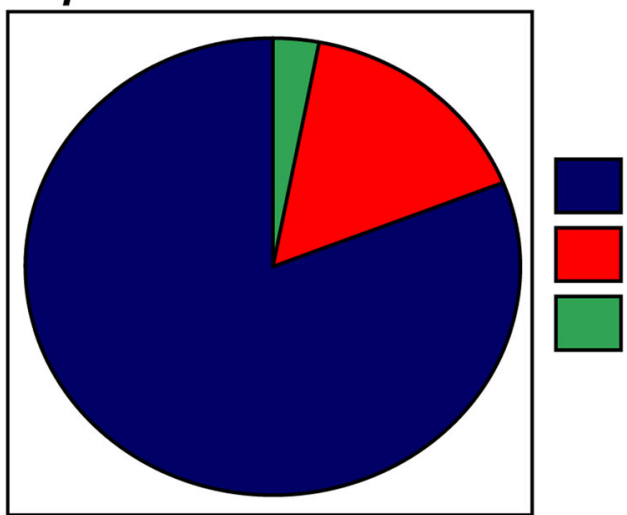

Copia $81 \%$

Gypsy $16 \%$

Unknown 3\%

\section{Copia}
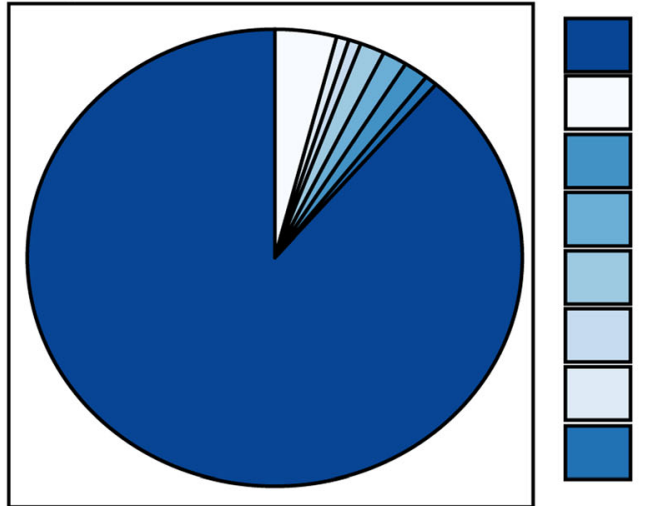

Ale $88.7 \%$

Tork $4.03 \%$

Bianca 1.6\%

Ivana 1.6\%

Ikeros $1.6 \%$

SIRE $0.8 \%$

TAR 0.8\%

Angela 0.8\%

\section{Gypsy}

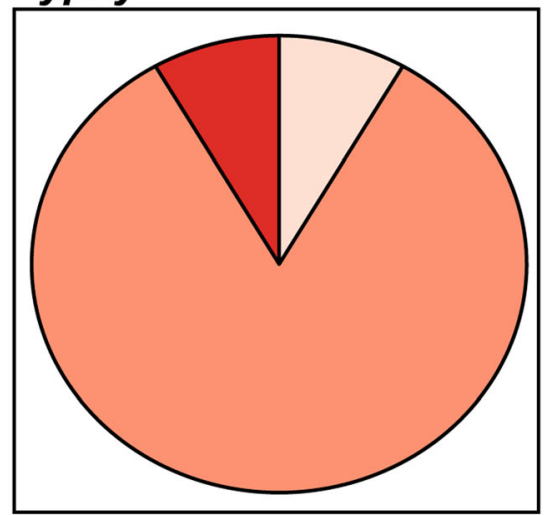

Chromovirus $83.3 \%$

Athila $8.3 \%$

Ogre-Tat $8.3 \%$

Fig. 5 Pie charts of expressed fig LTR-Res, considering both superfamilies and Copia and Gypsy lineage distributions

REs were analysed. Such correlations were not significant, probably because LTR-REs in a genome are a very heterogeneous population, made especially of lowly repeated and inactive elements. However, the measured regression lines can highlight trends in the relationships between LTR-RE abundance, insertion age and expression.

For example, the correlation between estimated insertion time and genome abundance (measured by average coverage) of Copia and Gypsy superfamilies was not significant; nevertheless, the regression lines suggested that older Copia LTR-REs were more abundant than younger ones in the F. carica genome, whereas Gypsy LTR-REs genome abundance was not different between young and old elements (Fig. 10).

Regarding the relationship between measured insertion time and expression level, LTR-REs belonging to the Gypsy superfamily were not analysed because they all showed very low RPKM values. The regression lines between age and expression level of full-length LTR-REs 


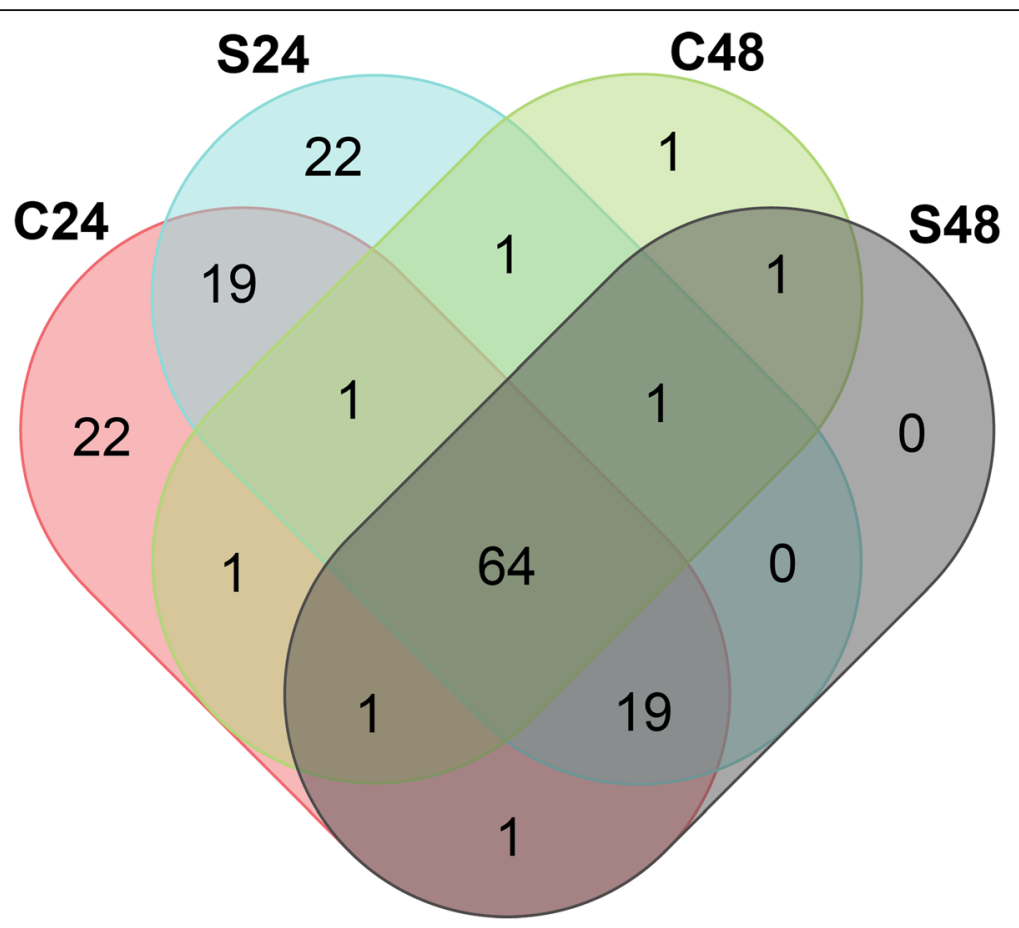

Fig. 6 Venn diagram of expressed LTR-REs after 24 and 48 days of salt treatment. $C=$ leaves from control plants, $S=$ leaves from salt treated plants

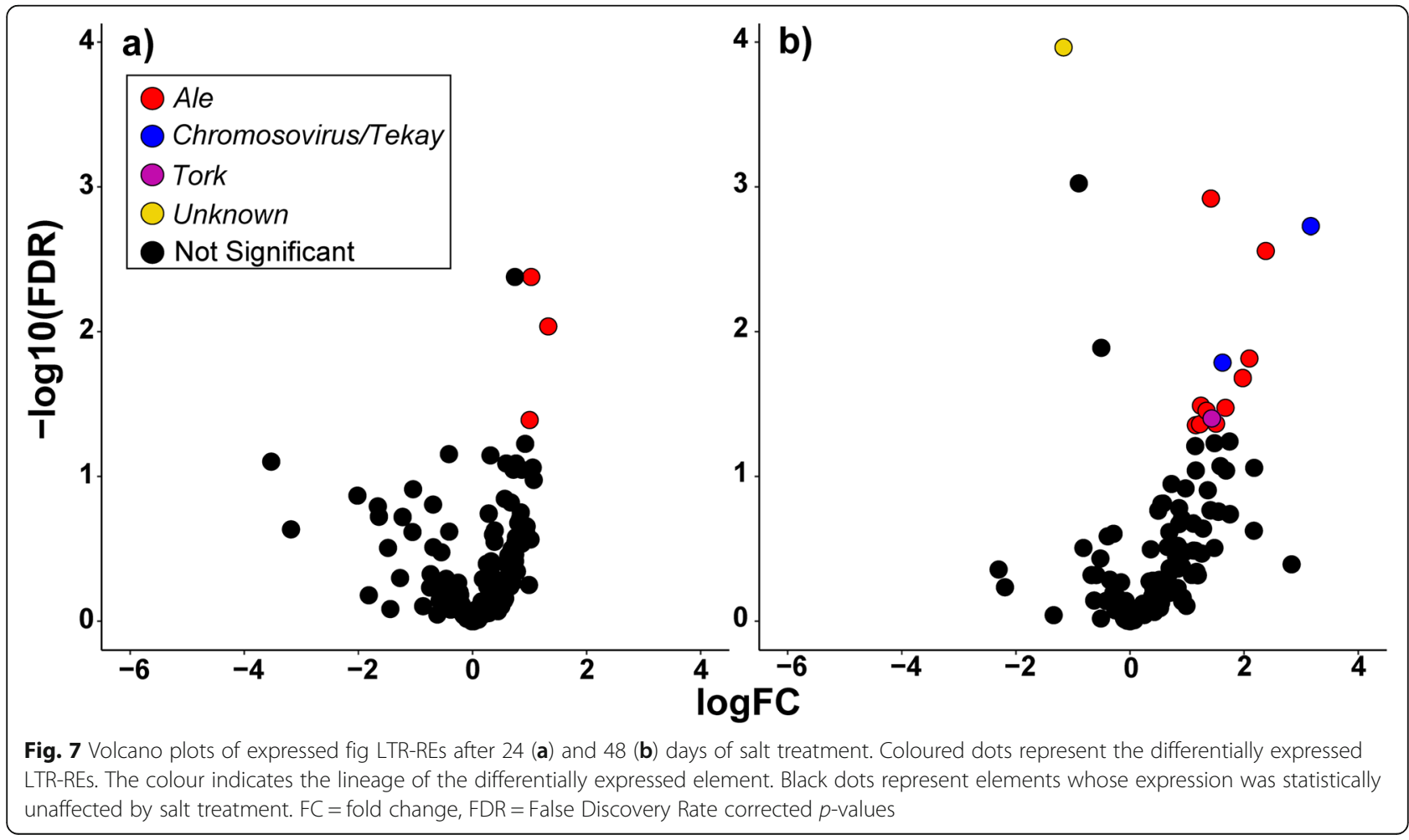



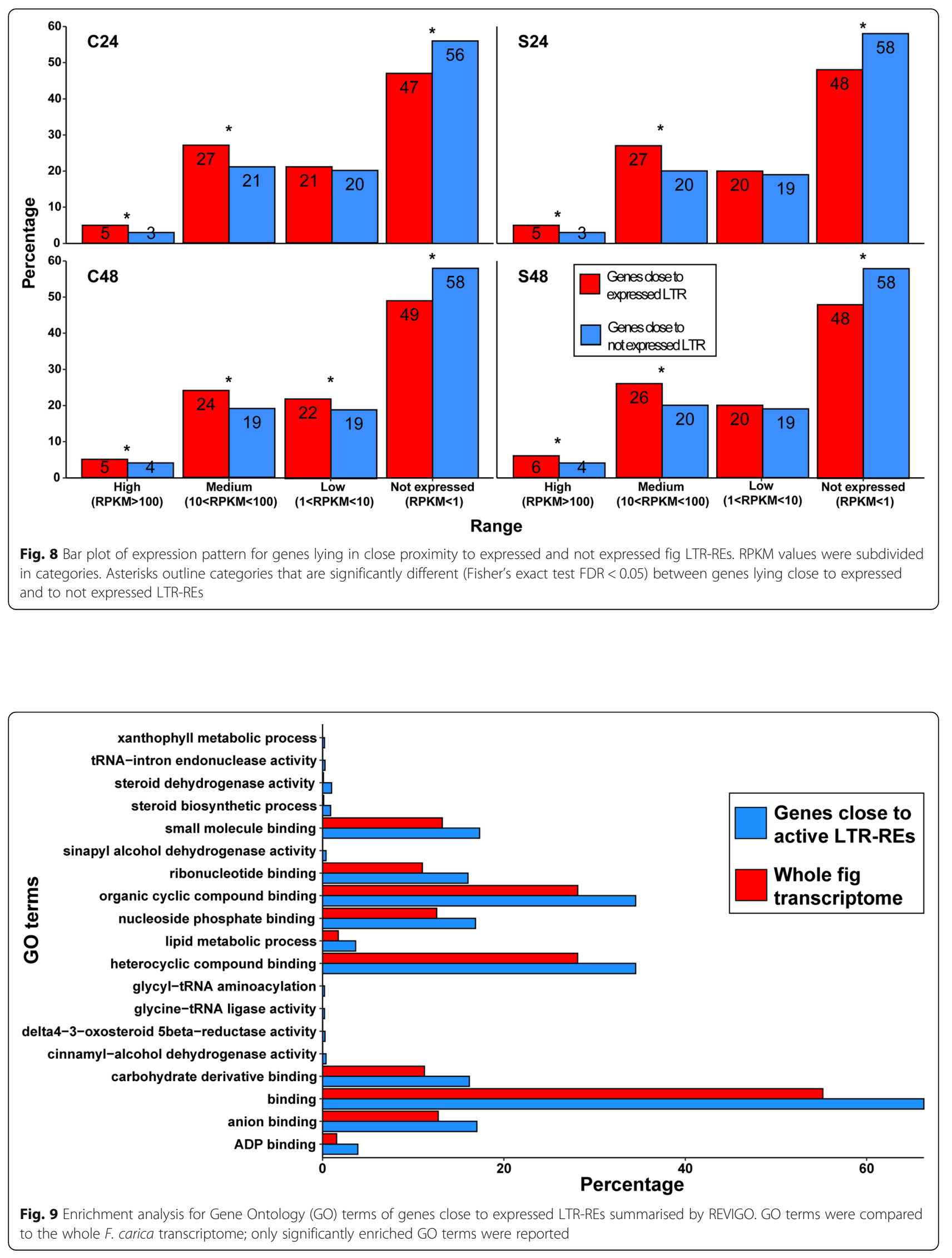

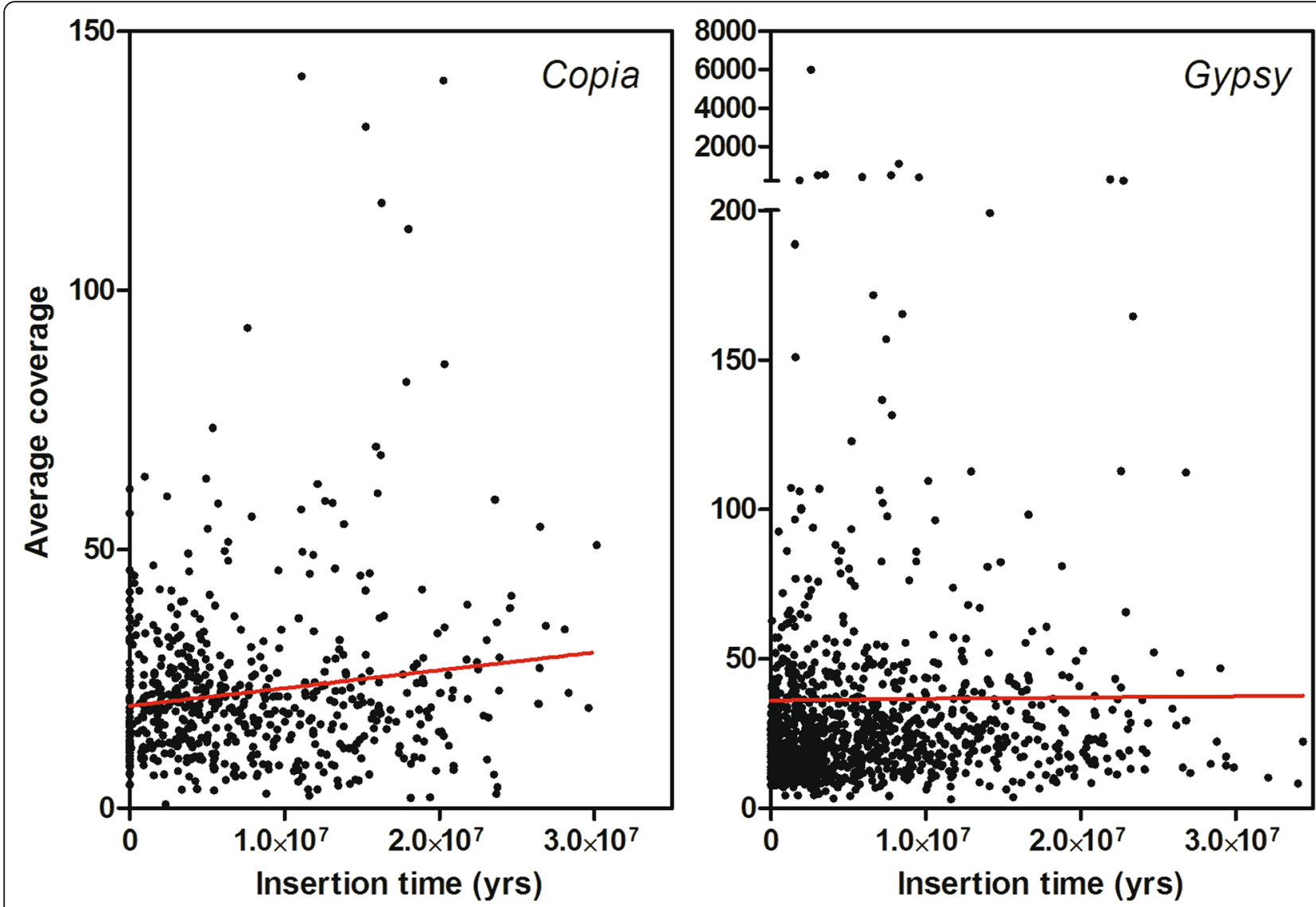

Fig. 10 Correlation between genomic abundance (reported as average coverage) and estimated insertion time (in years) for Copia and Gypsy fulllength LTR-REs in the F. carica genome

belonging to the Copia superfamily, in leaves of both control and salt-treated plants, showed that expression values tended to decrease with age (Fig. 11), although the correlation was not significant.

Finally, we analysed the correlation between genomic abundance (average coverage) and expression values (RPKM mean) of Copia full-length LTR-REs (Gypsy elements were not analysed because they were very lowly expressed). Although there was no statistical significance also in this correlation, regression lines outlined a trend according to which the more a LTR-RE was expressed, the less abundant (lowest average coverage) it was in the genome, in all treatments (Fig. 12).

In addition, by comparing the slopes of regression lines of the interaction estimated insertion time/expression level between control and salt-treated samples, we found that this value increased between control and saltstressed leaves both after 24 and 48 days since the beginning of the experiment (Table 1), suggesting possible activation of older LTR-REs during abiotic stress. Similarly, regression lines of genomic abundance against expression level showed an increase of the slopes during salt treatment after 24 and 48 days compared to control leaves. These data indicate the possible activation of most abundant full-length LTR-REs in the F. carica genome during saline treatment.

\section{Discussion}

The aim of our work was to provide complete characterisation of the LTR-REs of a species, F. carica, and their dynamics in the genome, through the study of all fulllength elements identifiable in the genome of this species. Analyses of the repeatome have been generally conducted using low coverage sequencing and clustering analyses (see for example, Mascagni et al. [36] and Zagorski et al. [37]) and rarely consider full-length elements, for example, in Populus trichocarpa [9] and Potentilla micrantha [10]. Nevertheless, the improvement of DNA sequencing and sequence assembling strategies that allow the use of long sequence reads are now achieving genome sequences with much greater completeness than before, especially in relation to the repetitive component of the genome. Therefore, genomes sequenced using these new procedures allow for a vastly more precise and reliable characterisation of repeated elements. 

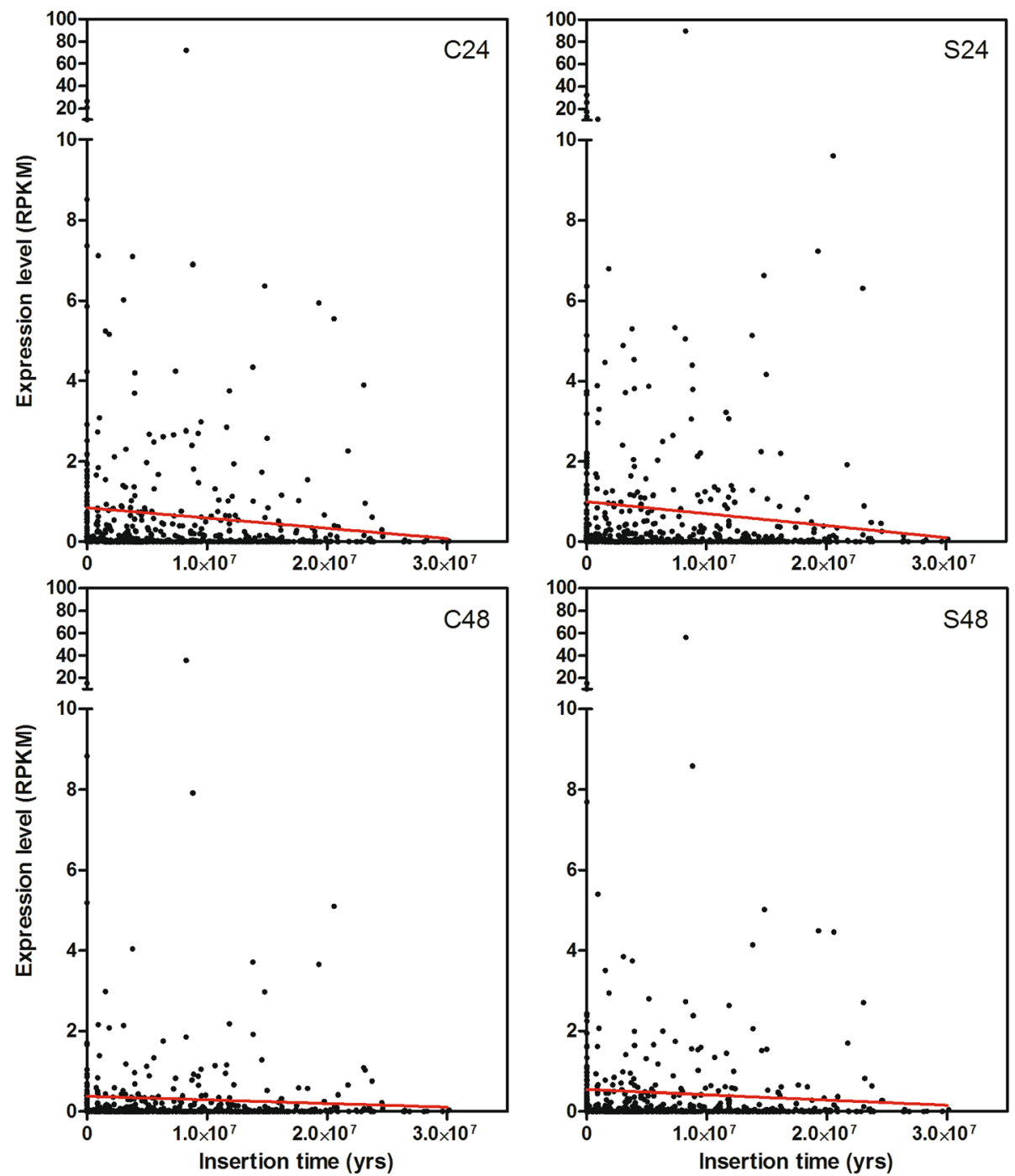

Fig. 11 Correlation between expression values, measured as RPKM mean and estimated insertion time (years) for Copia full-length LTR-REs in the F. carica genome

In the case described in this study, we analysed the genome of $F$. carica, obtained by combining PacBio and Illumina sequencing and recently released [1]. This species has a relatively small genome (356 Mbp [38]), and TEs represent roughly one third of the genome [1], a proportion similar to that found in other small-sized genomes [39] and higher than that measured on the previously available fig genome sequences (16\% [34]). This difference is most likely due to missing or collapsed repeated sequences, a typical phenomenon widely observed in assemblies generated through NGS technologies, especially when assemblies are highly fragmented. Other studies have already highlighted how longread technologies can successfully improve the resolution of repetitive genomic regions. For example, Oxford Nanopore Technology sequencing of Arabidopsis enabled the resolution of a quantitative trait loci (QTL) previously recalcitrant to $\mathrm{BAC}$ sequencing due to its repeat structure that required reads greater than $20 \mathrm{~kb}$ [40]. Other similar observations were highlighted in wheat [41] and maize [42].

In this study, we identified 1867 full-length elements that were classified in relation to their superfamily and lineage. The vast majority of these elements were specific to the PacBio-based assembly, confirming the higher quality of third generation sequencing technology based assemblies to resolve the complex repeated regions of a genome.

The full-length elements of the Gypsy superfamily were much more frequent than Copia, similarly to other species, such as poplar [9]. At the lineage level, for the Copia superfamily, Ale elements were the most frequent, 

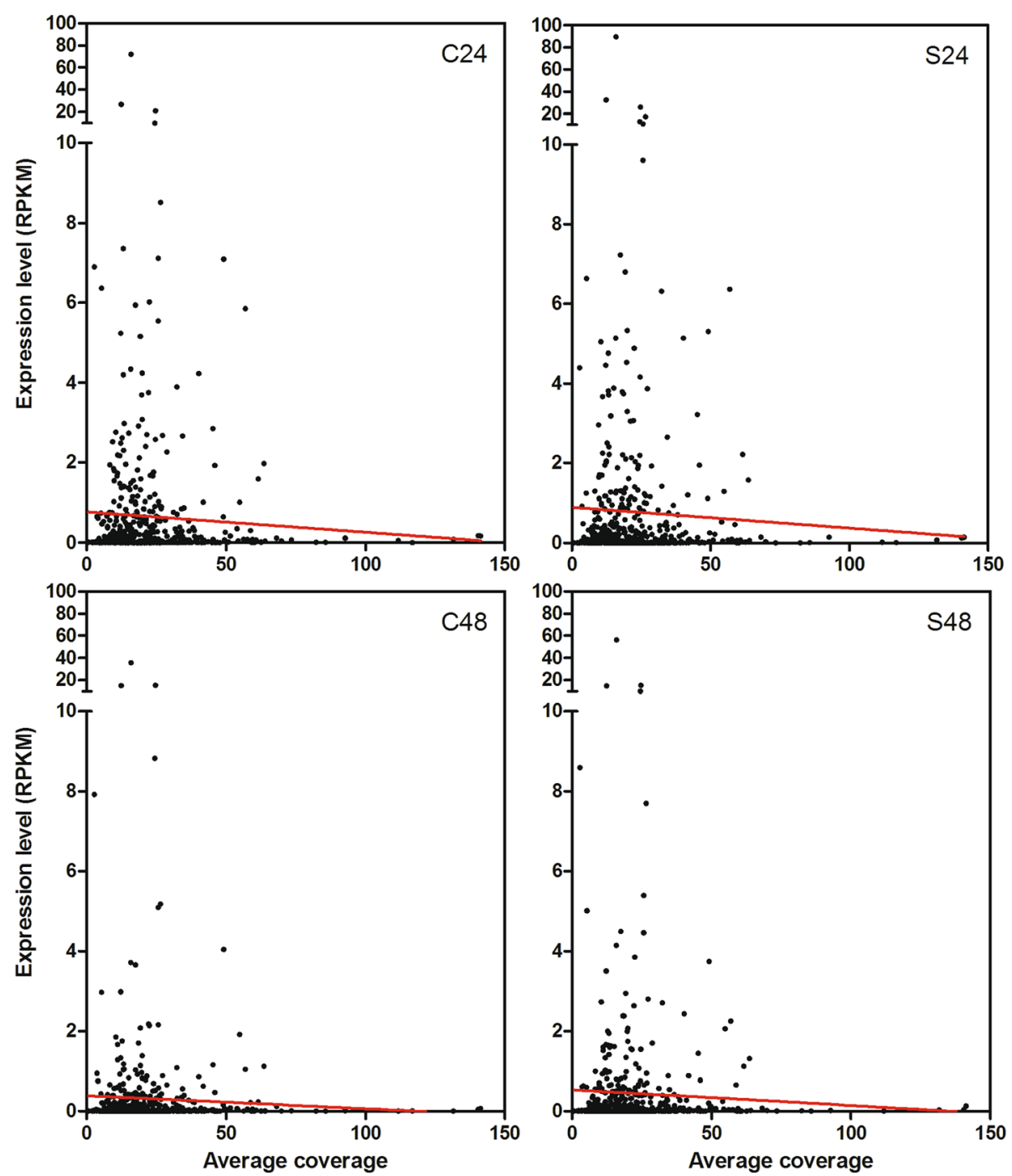

Fig. 12 Correlation between expression level (reported as RPKM mean) and genome abundance (reported as average coverage) for Copia fulllength LTR-REs in the F. carica genome

Table 1 Regression line slopes for each control (24 d and $48 \mathrm{~d}$, C24 and (48) and salt (24 d and $48 \mathrm{~d}$, S24 and S48) treatment in the correlations of expression level vs. estimated insertion time or vs. genome abundance of fig full-length LTR-REs

\begin{tabular}{lll}
\hline Correlation & Treatment & Slope \\
\hline Expression level / Insertion time & C24 & $-10,000$ \\
& S24 & -78.395 \\
& C48 & -129.900 \\
Expression level / Genome abundance & S48 24 & -90.595 \\
& S24 & -0.11 \\
& C48 & -0.075 \\
& S48 & -0.25 \\
&
\end{tabular}

similar to other species, such as Erythrostemon hughesii [43]. For the Gypsy superfamily, the Chromovirus lineage (in particular, the Tekay sublineage) showed the highest abundance as already reported in other species, like pepper and Hieracium [37, 44].

For each full-length element we measured the genome abundance, putative age of insertion, and transcription activity in leaves of control plants and of plants subjected to salt stress [33].

The Chromovirus lineage (sublineage CRM) showed the highest mean average coverage. Chromovirus/CRM elements are mainly located in the centromeres and in the pericentromeric regions, where they probably play a structural role [45-49]. Notably, the full-length elements of this sublineage were about $6 \%$ of all full-length elements identified in this work, while their mean average 
coverage (141.76) was much higher than those of all other lineages (ranging from 30.50 to 19.59), indicating the occurrence, in the fig genome, of many Chromovirus/CRM remnants and fragments (i.e., incomplete elements) not recognisable by the software that predicts only full-length LTR-REs, and probably related to the insertion of new elements within other elements (the socalled nested retrotransposons), structures that have already been described in the centromeric regions of other species [50].

Analysis of the insertion age of the full-length elements shows an extreme variability within each lineage, indicating wide insertion time spans, with large overlaps of activity for different elements, as already reported in poplar, sunflower, and mulberry $[9,51,52]$. On average, among the Copia elements, the lineages Ikeros and Tork were significantly older than lineages Ale and Ivana. Among Gypsy LTR-REs, the average age of the Chromovirus/CRM and Ogre elements was significantly higher than the Chromovirus/Tekay elements.

The expression of full-length elements was investigated by mapping Illumina cDNA reads obtained from leaves of plants subjected to salt stress and of control plants on the elements. In the case of repeated sequences, this type of analysis is biased because it is not certain that a cDNA read that maps to a full-length LTR-RE was not actually produced by an RNA transcribed from another element (or from an incomplete element) of the same family, which therefore shares its sequence with the full-length element and is located at another locus. However, the very high percentages of unique reads that mapped on the full-length LTR-REs (from 91.8 to $95.6 \%$ ) allowed us to establish reliable trends in the expression patterns of each element.

Our results showed that the expression of LTR-REs is generally very low, in both control and salt-exposed plants. This finding was expected because LTR-REs are commonly subjected to strict control that prevents their expression through different mechanisms. One major mechanism for transposon repression is RNA interference, which is mediated by small RNAs originating from double-stranded RNAs produced by the host, which trigger retrotransposon silencing through DNA methylation, chromatin remodelling, and post-transcriptional degradation of RE transcripts [53-55].

Considering RPKM $>1$ in at least one library as a threshold for defining a given expressed element, only 153 of 1867 elements were considered expressed in control and salt treated plants. Although Gypsy elements represented the vast majority of LTR-RE complements in the fig genome, most expressed elements belonged to the Copia superfamily. Qiu and Ungerer [56] found similar results in three wild species of the Helianthus genus, in which the most expressed LTR-REs belonged to the Copia superfamily and especially to barely repeated families. As a matter of fact, Gypsy elements are known to insert especially into heterochromatin [5759], while Copia LTR-REs tend to insert also into euchromatin regions [58, 60], that are more commonly subjected to transcription.

The presence of RE transcripts in plant tissues have been described in many species, without an apparent induction stimulus or following exposure to environmental changes [61]. The first case has been reported in species like Citrus sinensis, sunflower, rice, and poplar [62-66]. In the second group, induction of LTR-RE transcription has been shown when mimicking abiotic and biotic stress [67-74]. Notably, the occurrence of transcripts complementary to a LTR-RE sequence can be considered as i) the first step of LTR-RE activation, which may lead to final retrotransposition or ii) it may represent the production of LTR-RE RNAs to be used for RE silencing.

Concerning the expression of different LTR-RE lineages, the vast majority (88.7\%) of the expressed Copia elements belonged to the lineage Ale, and in the Gypsy superfamily, one lineage (Chromovirus) accounted for 83.3\% of expressed LTR-REs.

In general, salt stress showed little effect on the expression of LTR-REs. During early salt treatment, only three elements (all the Copia/Ale lineage) were upregulated. Ale elements were also the most represented among those (in total 13 elements) showing significant upregulation after 48 days of exposure to salt. Notably, the elements upregulated after 24 days were not the same as those upregulated after 48 days, suggesting a different type of induction by salt stress for elements of the same lineage.

Using RNA-seq data of leaves of salt-exposed and control plants, after 48 days, we analysed the effects of the presence of a LTR-RE on the expression of genes located in proximity to it (within $50 \mathrm{kbp}$ upand downstream to each full-length LTR-RE). We observed that most of the genes close to LTR-REs were not expressed. However, the expression of genes close to a LTR-RE was greater when the LTR$\mathrm{RE}$ was expressed than when it was not expressed. This data suggests a reciprocal influence between transposons and genes regarding transcription when they are close together on the chromosome. Such influence is probably linked to epigenetic control (for example, through DNA methylation) of the chromosomal locus, in which genes and LTR-REs are in proximity to each other. Methylation spreading from plant retrotransposons into flanking DNA regions has been reported [75, 76].

It is possible that the insertion of a retrotransposon could imply epigenetic control of expression in its 
adjacent regions, with consequent repression of transcription along that chromosomal locus [75]. In particular, it was shown that transposable element insertion can modify gene expression pattern even in the frame of thousands base pairs upstream, such as in the case the teosinte branched 1 gene of maize, whose expression pattern was modified by the insertion of a hopscotch retrotrasposon at a distance of more than $60,000 \mathrm{bp}$ from the gene [77].

However, it is also possible to hypothesise that, in the case of retrotransposon insertion in the proximity of genes whose expression is necessary to the cell and should be maintained, it may make it more difficult to silence that locus by DNA methylation. In this case, other LTR-RE silencing mechanisms should operate, probably on a post-transcriptional level [78].

By analysing the genes that are located close to expressed LTR-REs, we observed that some GOs are significantly more frequent in genes occurring in regions where expressed elements are found, indicating that the chromosomal regions where these genes are found are probably more difficult to be silenced by DNA methylation. Obviously, other studies are needed, especially in other species, to assess whether there is a relationship of this type between retrotransposons and certain functional categories of genes.

We then tried to evaluate possible correlations between genome abundance, insertion age, and expression of LTR-REs. The resulting regression lines were not significant, probably due to the extreme variability among LTR-REs regarding the insertion age and abundance. Such variability is related to the fact that each LTR-RE can be autonomous in replication in the host genome. Concerning the expression, only a few elements were expressed and the level of expression was generally very low, making it difficult to establish significant correlations. However, the regression lines obtained by our data allowed us to establish trends in LTR-RE dynamics.

Concerning the correlation between genome abundance and insertion age, for the Copia superfamily, the oldest elements were also more abundant indicating that, over time, there was continuous production of new copies of each element or that ancient LTR-REs were subjected to a replication burst. In contrast, for the Gypsy superfamily, it can be hypothesised that there is stronger control over the replication of the oldest elements, so that their abundance was not greater than that of the youngest ones. Cases have been reported in which even single elements were found to have had bursts of replication activity, such as to increase their genomic abundance in a short time span, in a range of species that include Vicia pannonica [79], cotton [80], Oryza australiensis [81], and Helianthus agrestis [66].
For the correlation between expression and age of insertion, it was possible to evaluate only the Copia elements, as the expression of the Gypsy elements was too small to allow a correlation study. This is another indication of the greater control that the host puts in place on this superfamily of elements than on the Copia superfamily.

Considering Copia elements, the expression tended to decrease as the insertion age increased, probably because the host had more time to set up epigenetic control of that element.

The same trend was observed for the correlation between the expression of LTR-REs and their genomic abundance. As a matter of fact, such a relationship was ascertained in several studies, in which the more an element was abundant the more it was recognised and subjected to silencing $[15,65,82,83]$.

\section{Conclusions}

In conclusion, our data provide a complete characterisation of the full-length LTR-REs of F. carica, yielding interesting information on the genomic dynamics related to these elements and on their role in gene regulation. The extension of this type of analysis to other species whose genome has been sequenced using thirdgeneration sequencing technologies will allow, through comparative analyses, a better understanding of the importance of these elements in the evolution of species at the genomic level.

\section{Methods}

Full-length LTR-REs isolation and characterisation

Class I full-length LTR-REs were identified in the highly contiguous fig genome sequence produced by Usai et al. [1] using the PacBio SMRT sequencing technology in combination with Illumina sequencing.

The elements were identified using LTRharvest v1.5.10 [84] and annotated using LTRdigest v1.5.10 [85] and the DANTE tool v1.0.0 provided on the RepeatExplorer Galaxy-based website (https://repeatexplorer-elixir.ceritsc.cz/galaxy/). LTRharvest was run on the fig genome assembly with the following parameters: -minlenltr 100 , -maxlenltr 6000, -mindistltr 1500, -maxdistltr 25,000, -mintsd 5, -maxtsd 5, -similar 85, -vic 10.

The available NGS-based fig genome sequence [34] was also subjected to the same structural-based identification process and a sequence comparison was performed by using $\mathrm{CD}$-Hit est. with similarity threshold set to $90 \%$ [86].

tRNA sequences of Arabidopsis thaliana, Populus trichocarpa, Vitis vinifera, and Zea mays were retrieved from GtRNAdb (http://gtrnadb.ucsc.edu) for the identification of the primer binding site regions of the identified elements through LTRdigest. 
The library of full-length LTR-REs was submitted to the DANTE tool for domain-based annotation. The process of annotation was run with default parameters using REXdb for transposable element protein domains [48] and a BLOSUM80 scoring matrix. The results were filtered by the significance of the protein matches with default parameters. Final annotation was manually checked to remove any nested cases.

In further characterisation of full-length elements, the insertion date of each LTR-RE was estimated through a pairwise sequence divergence comparison of the $5^{\prime}$ - and $3^{\prime}$-LTRs. LTR pairwise alignments and distance matrices were calculated using stretcher and distmat tools of the EMBOSS v6.6.0.0 suite, respectively [87], using the Kimura two-parameter model of sequence evolution [88]. The element insertion times were estimated using a mutation rate of $2.36 \times 10^{-9}$, i.e., two-fold the rate calculated for synonymous substitutions in gene sequences in Populus trichocarpa [89], because LTR-REs accumulate more mutations with time compared to gene sequences.

In some cases, the occurrence of protein encoding domains within an element was checked by using Augustus [90] and DANTE under default parameters.

\section{LTR-REs redundancy analysis}

DNA libraries of $F$. carica cv. Dottato were downloaded from the NCBI database (NCBI, Washington, USA, https://www.ncbi.nlm.nih.gov/sra) using accession number SRP109082. The construction of libraries was described by Solorzano Zambrano et al. [91]. DNA sequencing was performed with two Illumina sequencers: MiSeq and HiSeq2000, yielding 125 bp reads. The reads were trimmed using Trimmomatic v0.33 [92] with the following criteria: HEADCROP:19, CROP:100, SLIDINGWINDOW:4:20, MINLEN:100. Illumina adapters were also removed.

High-quality reads were cleaned from organellar sequences by mapping them to the $F$. carica chloroplast and Morus notabilis mitochondrial genomes using CLC Genomics Workbench v9.5.3 (CLC-BIO, Aarhus, Denmark) with stringent parameters: mismatch cost $=1$, deletion cost $=1$, insertion cost $=1$, similarity fraction $=$ 0.9 , and length fraction $=0.9$. The resulting un-mapped reads were collected.

The genomic abundance of LTR-REs in F. carica genome was assessed by aligning Illumina DNA reads to the full-length LTR-REs isolated from the assembly [1]. Mapping of Illumina DNA reads to full-length LTR-REs was performed using the CLC Genomics Workbench with the following criteria: mismatch cost $=1$, deletion $\operatorname{cost}=1$, insertion $\cos t=1$, similarity fraction $=0.9$, and length fraction $=0.9$.

\section{RNA isolation}

Two-year old F. carica (cv. Dottato), micropropagated plants were grown in $5 \mathrm{~L}$ plastic pots filled with a mixture of $6.4 \%$ clay, $8.6 \%$ silt, and $85 \%$ sand and subjected to salinity stress [33]. Plants were treated with salinity concentrations of 0 and $100 \mathrm{mM} \mathrm{NaCl}$, and two time points corresponding to 24 and 48 days after the beginning of the experiment were chosen. Hence, for each time point, one fully expanded leaf from three plants of the control $(0 \mathrm{mM} \mathrm{NaCl})$ and three salt-stressed plants $(100 \mathrm{mM} \mathrm{NaCl})$ were collected.

Total RNA was extracted from leaves according to Giordani et al. [65] and genomic DNA contamination removed by DNAse I (Roche) digestion following the manufacturer's instructions. Afterwards, RNA was purified following standard procedures. RNA-seq analyses were performed on control leaves $(0 \mathrm{mM} \mathrm{NaCl})$ and on leaves of plants treated with $100 \mathrm{mM} \mathrm{NaCl}$.

\section{LTR-REs and gene expression analyses}

RNA-seq analyses were performed on control leaves $(0$ $\mathrm{mM} \mathrm{NaCl}$ ) and on leaves of plants treated with $100 \mathrm{mM}$ $\mathrm{NaCl}$ at 24 and 48 days after the beginning of the experiment. Twelve cDNA libraries of control and stressed leaves of $F$. carica, obtained as described by Vangelisti et al. [33], were downloaded from NCBI SRA at BioProject accession code PRJNA508874.

The quality of each library was assessed by Bioanalyzer 2100 (Agilent Technologies, Santa Clara, CA, USA) and sequencing was performed by using Illumina HiSeq2000. The quality of the paired-end (125 bp length) read sequences was checked with FastQC (v. 0.11.5 [93] and trimmed with Trimmomatic, cropping the first 15 bases and last 10 bases and removing adapters.

cDNA reads were mapped to whole transcriptome and full-length LTR-REs by using the CLC Genomics Workbench with the same parameters described for DNA alignment. The expression level of LTR-REs was calculated as reads per kilo base per million mapped reads (RPKM, [94]); RPKM is a normalized value of expression which allows to compare transcription of sequences within and among libraries. RPKM is calculated by counting the reads aligned on a sequence on the total number of reads in a library, and on the sequence length [94]. Only LTR-REs with RPKM $>1$ in at least one library were considered expressed. To detect possible differentially expressed LTR-REs between the control and salt stressed leaves during the two time points, a pairwise likelihood test on edgeR [95] was applied to read counts derived from alignment. A False Discovery Rate (FDR) [96] was used for the resulting $p$-values. We considered those LTR-REs that showed an absolute FoldChange $>2$ and FDR $<0.05$ as differentially expressed. 
To evaluate the expression pattern of genes close to LTR-REs, the area in the $50 \mathrm{kbp}$ up- and down-stream of transposable elements was investigated. Genes in close proximity to these elements were extracted by using coordinates provided by the GFF file of the $F$. carica genome [1] and exploiting the intersect function of BEDtools v2.29.2 [97]. Expression values for genes in close proximity to LTR-REs were measured in terms of RPKM. These genes were split in classes by the range of RPKM. Then, expression was compared between genes lying in close proximity to expressed and not expressed LTR-REs. Differences were subjected to a Fisher's exact test and considered significant for FDR $<0.05$.

Functional analysis of genes was conducted by analysing GO terms provided with the $F$. carica gene annotation table [1]. The overall distribution of GO terms was visualised with WEGO 2.0 [98]. Blast2GO was used to perform enrichment analysis between the set of close genes to expressed LTR-REs and the whole transcriptome by using Fisher's exact test [99]; GO terms were selected as significant for FDR corrected $p$-values $<0.05$. Enriched GO terms were further summarised using REVIGO [100] by the tiny parameter option.

\section{Abbreviations}

FDR: False Discovery Rate; LTR: Long Terminal Repeat; LTR-RE: Long Terminal Repeat-Retrotransposon; MY: Million Years; PacBio: Pacific Biosciences; RE: Retrotransposon; RPKM: Reads Per Kilo base per Million mapped reads; SMRT: Single-Molecule Real-Time; TGS: Third-Generation-Sequencing

\section{Supplementary Information}

The online version contains supplementary material available at https://doi. org/10.1186/s12870-021-02991-x.

Additional file 1: Table S1. Number of mapped read onto full-length LTR-REs for each library of cDNA of control (C) and salt treated (S) plants after 24 and 48 days of treatment.

Additional file 2: Table S2. Genes which showed a stable expression pattern in control (C) and stressed leaves (S) during 24 and 48 days. Only genes that showed a low/medium expression similar to LTR-REs are reported.

Additional file 3: Figure S1. Box plot of RPKM values of expression for LTR-REs in F. carica leaves. The four box plots represent the expression level in leaves of the control (C) and salt treated (S) plants after 24 and 48 days since the beginning of the experiment.

\section{Acknowledgements}

Part of the computational resources used for this article were provided by the ELIXIR-CZ project (LM2015047), part of the international ELIXIR infrastructure.

\section{Authors' contributions}

$T G, L N$, and $A C$ conceived the research; $A V, F M$, and GU designed the experiments; AV, SS, MV, and GU carried out the experiments and analysed the data; AC, FM, AV, SS, LN, and TG wrote the manuscript. All authors have read and approved the final manuscript.

\section{Funding}

This study was performed in the frame of FIGGEN/PRIMA19_00197 project, that is part of the PRIMA Programme supported by the European Union through a national MIUR (Italy) grant.

\section{Availability of data and materials}

DNA sequencing data have been deposited in the NCBI repository under BioProject accession code PRJNA565858. The fig genome has been deposited at DDBJ/ENA/GenBank under the accession VYVB00000000. The version described in this paper is version WYB01000000. The collection of TEs is available at the repository sequence page of the Department of Agriculture, Food, and Environment of the University of Pisa (http://pgagl.agr unipi.it/sequence-repository/). RNA sequencing data have been deposited in the NCBI repository under Bioproject accession code PRJNA508874.

Intermediary files and other miscellaneous information are available from the corresponding author on reasonable request.

Ethics approval and consent to participate

Not applicable.

\section{Consent for publication}

Not applicable.

\section{Competing interests}

The authors declare that they have no conflict of interest.

Received: 24 December 2020 Accepted: 15 April 2021

Published online: 17 May 2021

\section{References}

1. Usai G, Mascagni F, Giordani T, Vangelisti A, Bosi E, Zuccolo A, et al. Plant J. 2020;102:600-14.

2. Barolo MI, Mostacero NR, López SN. Ficus carica L.(Moraceae): an ancient source of food and health. Food Chem. 2014;164:119-27. https://doi.org/1 0.1016/j.foodchem.2014.04.112

3. Barghini E, Mascagni F, Giordani T, Solorzano Zambrano LJ, Natali L, Cavallini A. An insight into structure and composition of the fig genome. Acta Hortic. 2017:1173:69-74.

4. Tapia R, Botti C, Carrasco O, Prat L, Franck N. Effect of four irrigation rates on growth of six fig tree varieties. Acta Hortic. 2003;605:113-8.

5. Ayers SR, Westcost WD. Water quality for agriculture, FAO irrigation and drainage paper 29. Rome, Italy: Food and agriculture Organization of the United Nations; 1979.

6. Caruso G, Gennai C, Ugolini F, Marchini F, Quartacci MF, Gucci R. Tolerance and physiological response of young Ficus carica L. plants irrigated with saline water. Acta Hortic. 2017;1173:137-42.

7. Wicker T, Sabot F, Hua-Van A, Bennetzen JL, Capy P, Chalhoub B, et al. A unified classification system for eukaryotic transposable elements. Nature Rev Genet. 2007:8(12):973-82. https://doi.org/10.1038/nrg2165.

8. Kumar A, Bennetzen JL. Plant retrotransposons. Ann Rev Genet. 1999;33(1): 479-532. https://doi.org/10.1146/annurev.genet.33.1.479.

9. Natali L, Cossu RM, Mascagni F, Giordani T, Cavallini A. A survey of Gypsy and Copia LTR-retrotransposon superfamilies and lineages and their distinct dynamics in the Populus trichocarpa (L.) genome. Tree Genet Genomes. 2015;11:107.

10. Buti M, Moretto M, Barghini E, Mascagni F, Natali L, Brilli M, et al. The genome sequence and transcriptome of Potentilla micrantha and their comparison to Fragaria vesca (the woodland strawberry). GigaScience. 2018; 7(4):1-14. https://doi.org/10.1093/gigascience/giy010.

11. Ma J, Devos KM, Bennetzen JL. Analyses of LTR-retrotransposon structures reveal recent and rapid genomic DNA loss in rice. Genome Res. 2004;14(5): 860-9. https://doi.org/10.1101/gr.1466204.

12. Wang Q, Dooner HK. Remarkable variation in maize genome structure inferred from haplotype diversity at the bz locus. Proc Natl Acad Sci U S A. 2006;103(47):17644-9. https://doi.org/10.1073/pnas.0603080103.

13. Devos KM, Brown JK, Bennetzen JL. Genome size reduction through illegitimate recombination counteracts genome expansion in Arabidopsis. Genome Res. 2002;12(7):1075-9. https://doi.org/10.1101/gr.132102.

14. Vitte C, Panaud O. Formation of solo-LTRs through unequal homologous recombination counterbalances amplifications of LTR retrotransposons in rice Oryza sativa L. Mol Biol Evol. 2003;20(4):528-40. https://doi.org/10.1093/ molbev/msg055.

15. Lisch D. How important are transposons for plant evolution? Nature Rev Genet. 2013;14(1):49-61. https://doi.org/10.1038/nrg3374.

16. Pinosio S, Giacomello S, Faivre-Rampant P, Taylor G, Jorge V, Le Paslier MC, et al. Characterization of the poplar pan-genome by genome-wide 
identification of structural variation. Mol Biol Evol. 2016;33(10):2706-19. https://doi.org/10.1093/molbev/msw161.

17. Dubin MJ, Scheid OM, Becker C. Transposons: a blessing curse. Curr Opinion Plant Biol. 2018:42:23-9. https://doi.org/10.1016/j.pbi.2018.01.003.

18. Van Driel R, Fransz PF, Verschure PJ. The eukaryotic genome: a system regulated at different hierarchical levels. J Cell Sci. 2003;1 16(20):4067-75. https://doi.org/10.1242/jcs.00779.

19. Song JT, Lu H, McDowell JM, Greenberg JT. A key role for ALD1 in activation of local and systemic defenses in Arabidopsis. Plant J. 2004;40(2): 200-12. https://doi.org/10.1111/j.1365-313X.2004.02200.x.

20. Hollister JD, Gaut BS. Epigenetic silencing of transposable elements: a tradeoff between reduced transposition and deleterious effects on neighboring gene expression. Genome Res. 2009;19(8):1419-28. https://doi.org/10.1101/ gr.091678.109.

21. Hollister JD, Smith LM, Guo YL, Ott F, Weigel D, Gaut BS. Transposable elements and small RNAs contribute to gene expression divergence between Arabidopsis thaliana and Arabidopsis lyrata. Proc Natl Acad Sci U S A. 2011;108(6):2322-7. https://doi.org/10.1073/pnas.1018222108.

22. Liu J, Seetharam AS, Chougule K, Ou S, Swentowsky KW, Gent Jl, et al. Gapless assembly of maize chromosomes using long-read technologies. Genome Biol. 2020;21:1-17.

23. Matzke MA, Mosher RA. RNA-directed DNA methylation: an epigenetic pathway of increasing complexity. Nature Rev Genet. 2014;15(6):394-408. https://doi.org/10.1038/nrg3683.

24. Bucher E, Reinders J, Mirouze M. Epigenetic control of transposon transcription and mobility in Arabidopsis. Curr Opinion Plant Biology. 2012; 15(5):503-10. https://doi.org/10.1016/j.pbi.2012.08.006.

25. Casacuberta E, González J. The impact of transposable elements in environmental adaptation. Mol Ecology. 2013;22(6):1503-17. https://doi. org/10.1111/mec.12170.

26. Vangelisti A, Usai G, Mascagni F, Natali L, Giordani T, Cavallini A. A whole genome analysis of long-terminal-repeat retrotransposon transcription in leaves of Populus trichocarpa $L$ subjected to different stresses. Caryologia. 2019;72:69-79.

27. Vangelisti A, Mascagni F, Usai G, Natali L, Giordani T, Cavallini A. Low long terminal repeat (LTR)-retrotransposon expression in leaves of the marine phanerogam Posidonia oceanica L. Life. 2020;10(3):30. https://doi.org/10.33 90/life10030030

28. Ansorge WJ. Next generation DNA sequencing (II): techniques, applications. Next Generat Sequenc \& Applic. 2016;1:1-10.

29. Daccord N, Celton JM, Linsmith G, Becker C, Choisne N, Schijlen E, et al. High-quality de novo assembly of the apple genome and methylome dynamics of early fruit development. Nature Genet. 2017;49(7):1099-106. https://doi.org/10.1038/ng.3886.

30. Vogel A, Schwacke R, Denton AK, Usadel B, Hollmann J, Fischer K, et al. Footprints of parasitism in the genome of the parasitic flowering plant Cuscuta campestris. Nature Comm. 2018;9:1-11.

31. Ye G, Zhang H, Chen B, Nie S, Liu H, Gao W, et al. De novo genome assembly of the stress tolerant forest species Casuarina equisetifolia provides insight into secondary growth. Plant J. 2019;97(4):779-94. https://doi.org/1 $0.1111 /$ tpj.14159.

32. Veeckman E, Ruttink T, Van De Poele K. Are we there yet? Reliably estimating the completeness of plant genome sequences. Plant Cell. 2016; 28(8):1759-68. https://doi.org/10.1105/tpc.16.00349.

33. Vangelisti A, Solorzano Zambrano L, Caruso G, Macheda D, Bernardi R, Usai $\mathrm{G}$, et al. How an ancient, salt-tolerant fruit crop, Ficus carica L., copes with salinity: a transcriptome analysis. Sci Rep. 2019;9:1-13.

34. Mori K, Shirasawa K, Nogata H, Hirata C, Tashiro K, Habu T, et al. Identification of RAN1 orthologue associated with sex determination through whole genome sequencing analysis in fig (Ficus carica L.). Sci Rep. 2017;7:41124.

35. SanMiguel P, Gaut BS, Tikhonov A, Nakajima Y, Bennetzen JL. The paleontology of intergene retrotransposons of maize. Nature Genet. 1998; 20(1):43-5. https://doi.org/10.1038/1695.

36. Mascagni F, Vangelisti A, Giordani T, Cavallini A, Natali L. A computational comparative study of the repetitive DNA in the genus Quercus $\mathrm{L}$. Tree Genet Genomes. 2020;16(1):11. https://doi.org/10.1007/s11295-019-1401-2.

37. Zagorski D, Hartmann M, Bertrand YJ, Paštová L, Slavíková R, Josefiová J, et al. Characterization and dynamics of repeatomes in closely related species of Hieracium (Asteraceae) and their synthetic and apomictic hybrids. Front Plant Sci. 2020;11:591053. https://doi.org/10.3389/fpls.2020.591053.
38. Loureiro J, Rodriguez E, Dolezěl J, Santos C. Two new nuclear isolation buffers for plant DNA flow cytometry: a test with 37 species. Ann Bot. 2007; 100(4):875-88. https://doi.org/10.1093/aob/mcm152.

39. Vitte C, Fustier MA, Alix K, Tenaillon MI. The bright side of transposons in crop evolution. Brief Funct Genomics. 2014;13(4):276-95. https://doi.org/10.1 093/bfgp/elu002.

40. Michael TP, Jupe F, Bemm F, Motley ST, Sandoval JP, Lanz C, et al. High contiguity Arabidopsis thaliana genome assembly with a single nanopore flow cell. Nature Comm. 2018;9:1-8.

41. Zhang W, Ciclitira P, Messing J. PacBio sequencing of gene families - a case study with wheat gluten genes. Gene. 2014;533(2):541-6. https://doi. org/10.1016/j.gene.2013.10.009.

42. Wang B, Tseng E, Regulski M, Clark TA, Hon T, Jiao Y, et al. Unveiling the complexity of the maize transcriptome by single-molecule long-read sequencing. Nature Comm. 2016;7:1-13.

43. Mata-Sucre Y, Sader M, Van-Lume B, Gagnon E, Pedrosa-Harand A, Leitch IJ, et al. How diverse is heterochromatin in the Caesalpinia group? Cytogenomic characterization of Erythrostemon hughesii Gagnon \& GP Lewis (Leguminosae: Caesalpinioideae). Planta. 2020;252:1-14.

44. de Assis R, Baba VY, Cintra LA, Gonçalves LSA, Rodrigues R, Vanzela ALL. Genome relationships and LTR-retrotransposon diversity in three cultivated Capsicum L. (Solanaceae) species. BMC Genomics. 2020;21:1-14.

45. Sharma A, Presting GG. Centromeric retrotransposon lineages predate the maize/rice divergence and differ in abundance and activity. Mol Gen Genomics. 2008;279(2):133-47. https://doi.org/10.1007/s00438-007-0302-5.

46. Gong ZY, Wu YF, Koblizkova A, Torres GA, Wang K, lovene M, et al. Repeatless and repeat-based centromeres in potato: implications for centromere evolution. Plant Cell. 2012;24(9):3559-74. https://doi.org/10.11 05/tpc.112.100511.

47. Su HD, Liu YL, Liu YX, Lu ZL, Li HY, Xie SJ, et al. Dynamic chromatin changes associated with de novo centromere formation in maize euchromatin. Plant J. 2016;88(5):854-66. https://doi.org/10.1111/tpj.13305.

48. Neumann P, Novák P, Hoštáková N, Macas J. Systematic survey of plant LTRretrotransposons elucidates phylogenetic relationships of their polyprotein domains and provides a reference for element classification. Mob DNA. 2019:10(1):1. https://doi.org/10.1186/s13100-018-0144-1.

49. Kirov I, Odintsov S, Omarov M, Gvaramiya S, Merkulov P, Dudnikov M, et al. Functional Allium fistulosum centromeres comprise arrays of a long satellite repeat, insertions of retrotransposons and chloroplast DNA. Front Plant Sci. 2020;11:562001. https://doi.org/10.3389/fpls.2020.562001.

50. Weber B, Schmidt T. Nested Ty3-gypsy retrotransposons of a single Beta procumbens centromere contain a putative chromodomain. Chrom Res. 2009;17(3):379-96. https://doi.org/10.1007/s10577-009-9029-y.

51. Mascagni F, Vangelisti A, Giordani T, Cavallini A, Natali L. Specific LTRretrotransposons show copy number variations between wild and cultivated sunflowers. Genes. 2018;9(9):433. https:/doi.org/10.3390/genes9090433.

52. Ma B, Kuang L, Xin $Y$, He N. New insights into long terminal repeat retrotransposons in mulberry species. Genes. 2019;10(4):285. https://doi. org/10.3390/genes 10040285.

53. Slotkin RK, Martienssen R. Transposable elements and the epigenetic regulation of the genome. Nature Rev Genet. 2007;8(4):272-85. https://doi. org/10.1038/nrg2072.

54. Lisch D. Epigenetic regulation of transposable elements in plants. Ann Rev Plant Biol. 2009;60(1):43-66. https://doi.org/10.1146/annurev.arplant.59.032 607.092744.

55. Yang F, Wang PJ. Multiple LINEs of retrotransposon silencing mechanisms in the mammalian germline. In: Seminars in Cell \& Developmental Biology (Vol 59): Academic Press; 2016. p. 118-25.

56. Qiu F, Ungerer MC. Genomic abundance and transcriptional activity of diverse gypsy and copia long terminal repeat retrotransposons in three wild sunflower species. BMC Plant Biol. 2018;18:1-8.

57. Gao X, Hou Y, Ebina H, Levin HL, Voytas DF. Chromodomains direct integration of retrotransposons to heterochromatin. Genome Res. 2008; 18(3):359-69. https://doi.org/10.1101/gr.7146408.

58. Cavallini A, Natali L, Zuccolo A, Giordani T, Jurman I, Ferrillo V, et al. Analysis of transposons and repeat composition of the sunflower (Helianthus annuus L.) genome. Theor Appl Genet. 2010;120(3):491-508. https://doi.org/10.1007/ s00122-009-1170-7.

59. Novikov A, Smyshlyaev G, Novikova O. Evolutionary history of LTR retrotransposon chromodomains in plants. Int J Plant Genomics. Int J Plant Genomics. 2012;2012:874743. https://doi.org/10.1155/2012/874743. 
60. Pearce SR, Harrison G, Li D, Heslop-Harrison JS, Kumar A, Flavell AJ. The Ty1copia group retrotransposons in Vicia species: copy number, sequence heterogeneity and chromosomal localisation. Mol Gen Genet. 1996;250(3): 305-15. https://doi.org/10.1007/BF02174388.

61. Grandbastien MA. LTR retrotransposons, handy hitchhikers of plant regulation and stress response. Bioch Biophys Acta. 1849;2015:403-16.

62. Rico-Cabanas L, Martínez-Izquierdo JA. CIRE1, a novel transcriptionally active Ty1-copia retrotransposon from Citrus sinensis. Mol Gen Genomics. 2007; 277(4):365-77. https://doi.org/10.1007/s00438-006-0200-2.

63. Vukich M, Schulman AH, Giordani T, Natali L, Kalendar R, Cavallini A. Genetic variability in sunflower (Helianthus annuus L.) and in the Helianthus genus as assessed by retrotransposon-based molecular markers. Theor Appl Genet. 2009;119:1027-38

64. Gao D, Jiang N, Wing RA, Jiang J, Jackson SA. Transposons play an important role in the evolution and diversification of centromeres among closely related species. Frontiers Plant Science. 2015;6:216.

65. Giordani T, Cossu RM, Mascagni F, Marroni F, Morgante M, Cavallini A, et al. Genome-wide analysis of LTR-retrotransposon expression in leaves of Populusx canadensis water-deprived plants. Tree Genet Genomes. 2016; 12(4):75. https://doi.org/10.1007/s11295-016-1036-5.

66. Mascagni F, Giordani T, Ceccarelli M, Cavallini A, Natali L. Genome-wide analysis of LTR-retrotransposon diversity and its impact on the evolution of the genus Helianthus (L.). BMC Genomics. 2017;18(1):634. https://doi.org/1 0.1186/s12864-017-4050-6.

67. Kashkush K, Feldman M, Levy AA. Transcriptional activation of retrotransposons alters the expression of adjacent genes in wheat. Nature Genet. 2003:33(1):102-6. https://doi.org/10.1038/ng1063.

68. Hawkins JS, Hu G, Rapp RA, Grafenberg JL, Wendel JF. Phylogenetic determination of the pace of transposable element proliferation in plants: copia and LINE-like elements in Gossypium. Genome. 2008;51(1):11-8. https://doi.org/10.1139/G07-099.

69. He P, Ma Y, Dai H, Li L, Liu Y, Li H, et al. Characterization of the hormone and stress-induced expression of FaRE1 retrotransposon promoter in strawberry. J Plant Biol. 2012;55(1):1-7. https://doi.org/10.1007/s12374-0119180-9.

70. Ito H, Yoshida T, Tsukahara S, Kawabe A. Evolution of the ONSEN retrotransposon family activated upon heat stress in Brassicaceae. Gene. 2013;518(2):256-61. https://doi.org/10.1016/j.gene.2013.01.034.

71. Fan F, Cui B, Zhang T, Ding G, Wen X. LTR-retrotransposon activation, IRAP marker development and its potential in genetic diversity assessment of masson pine (Pinus massoniana). Tree Genet Genomes. 2014;10(1):213-22. https://doi.org/10.1007/s11295-013-0677-X.

72. Voronova A, Belevich V, Jansons A, Rungis D. Stress-induced transcriptional activation of retrotransposon-like sequences in the Scots pine (Pinus sylvestris L.) genome. Tree Genet Genomes. 2014;10:937-51.

73. Esposito S, Barteri F, Casacuberta J, Mirouze M, Carputo D, Aversano R. LTRTEs abundance, timing and mobility in Solanum commersonii and $S$. tuberosum genomes following cold-stress conditions. Planta. 2019;250(5): 1781-7. https://doi.org/10.1007/s00425-019-03283-3.

74. Mascagni F, Vangelisti A, Usai G, Giordani T, Cavallini A, Natali L. A computational genome-wide analysis of long terminal repeats retrotransposon expression in sunflower roots (Helianthus annuus L.). Genetica. 2020;148(1):13-23. https://doi.org/10.1007/s10709-020-00085-4

75. Arnaud P, Goubely C, Pelissier T, Deragon JM. SINE retroposons can be used in vivo as nucleation centers for de novo methylation. Mol Cell Biol. 2000; 20(10):3434-41. https://doi.org/10.1128/MCB.20.10.3434-3441.2000.

76. Saze H, Kakutani T. Heritable epigenetic mutation of a transposon-flanked Arabidopsis gene due to lack of the chromatin-remodeling factor DDM1. EMBO J. 2007;26(15):3641-52. https://doi.org/10.1038/sj.emboj.7601788.

77. Studer A, Zhao Q, Ross-Ibarra J, Dobley J. Identification of a functional transposon insertion in the maize domestication gene tb1. Nature Genet. 2011;43(11):1160-3. https://doi.org/10.1038/ng.942.

78. Sigman MJ, Slotkin RK. The first rule of plant transposable element silencing: location, location, location. Plant Cell. 2016;28(2):304-13. https://doi.org/1 $0.1105 /$ tpc.15.00869

79. Neumann P, Kobližková A, Navrátilová A, Macas J. Significant expansion of Vicia pannonica genome size mediated by amplification of a single type of giant retroelement. Genetics. 2006;173(2):1047-56. https://doi.org/10.1534/ genetics.106.056259.

80. Hawkins JS, Kim H, Nason JD, Wing RA, Wendel JF. Differential lineagespecific amplification of transposable elements is responsible for genome size variation in Gossypium. Genome Res. 2006;16(10):1252-61. https://doi. org/10.1101/gr.5282906.

81. Piegu B, Guyot R, Picault N, Roulin A, Saniyal A, Kim H, et al. Doubling genome size without polyploidization: dynamics of retrotransposition-driven genomic expansions in Oryza australiensis, a wild relative of rice. Genome Res. 2006;16(10):1262-9. https://doi.org/10.1101/gr.5290206.

82. Meyers BC, Tingey SV, Morgante M. Abundance, distribution, and transcriptional activity of repetitive elements in the maize genome. Genome Res. 2001;11(10):1660-76. https://doi.org/10.1101/gr.188201.

83. Yamazaki M, Tsugawa H, Miyao A, Yano M, Wu J, Yamamoto S, et al. The rice retrotransposon Tos 17 prefers low-copy-number sequences as integration targets. Mol Gen Genomics. 2001;265(2):336-44. https://doi.org/1 $0.1007 / \mathrm{s} 004380000421$

84. Ellinghaus D, Kurtz S, Willhoeft U. LTRharvest, an efficient and flexible software for de novo detection of LTR retrotransposons. BMC Bioinformatics. 2008;9(1):18. https://doi.org/10.1186/1471-2105-9-18.

85. Steinbiss S, Willhoeft U, Gremme G, Kurtz S. Fine-grained annotation and classification of de novo predicted LTR retrotransposons. Nucl Acids Res. 2009;37(21):7002-13. https://doi.org/10.1093/nar/gkp759.

86. Fu L, Niu B, Zhengwei Z, Sitao W, Weizhong L. CD-HIT: accelerated for clustering the next-generation sequencing data. Bioinformatics. 2012;28(23): 3150-2. https://doi.org/10.1093/bioinformatics/bts565.

87. Rice $\mathrm{P}$, Longden I, Bleasby A. EMBOSS: the European molecular biology open software suite. TIG. 2000;16(6):276-7. https://doi.org/10.1016/S01689525(00)02024-2.

88. Kimura M. A simple method for estimating evolutionary rates of base substitutions through comparative studies of nucleotide sequences. J Mol Evol. 1980;16(2):111-20. https://doi.org/10.1007/BF01731581.

89. Mascagni F, Usai G, Natali L, Cavallini A, Giordani T. A comparison of methods for LTR-retrotransposon insertion time profiling in the Populus trichocarpa genome. Caryologia. 2018;71(1):85-92. https://doi.org/10.1080/ 00087114.2018 .1429749 .

90. Stanke M, Mongersterne B. AUGUSTUS: a web server for gene prediction in eukaryotes that allows user-defined constraints. Nucl Acids Res. 2005; 33(Web Server):W465-7. https://doi.org/10.1093/nar/gki458.

91. Solorzano Zambrano L, Usai G, Vangelisti A, Mascagni F, Giordani T, Bernardi $\mathrm{R}$, et al. Cultivar-specific transcriptome prediction and annotation in Ficus carica L. Genomics Data. 2017;13:64-6. https://doi.org/10.1016/j.gdata.2017. 07.005 .

92. Bolger AM, Lohse M, Usadel B. Trimmomatic: a flexible trimmer for Illumina sequence data. Bioinformatics. 2014;30(15):2114-20. https://doi.org/10.1093/ bioinformatics/btu170.

93. Andrews S. FastQC: a quality control tool for high throughput sequence data; 2010. p. 370

94. Mortazavi A, Williams BA, McCue K, Schaeffer L, Wold B. Mapping and quantifying mammalian transcriptomes by RNA-Seq. Nature Meth. 2008;5(7): 621-8. https://doi.org/10.1038/nmeth.1226.

95. Robinson MD, McCarthy DJ, Smyth GK. edgeR: a bioconductor package for differential expression analysis of digital gene expression data. Bioinformatics. 2010;26(1):139-40. https://doi.org/10.1093/bioinformatics/ btp616.

96. Benjamini Y, Hochberg Y. Controlling the false discovery rate: a practical and powerful approach to multiple testing. J Royal Stat Soc Ser B. 1995;57: 289-300.

97. Quinlan AR, Hall IM. BEDTools: a flexible suite of utilities for comparing genomic features. Bioinformatics. 2010;26(6):841-2. https://doi.org/10.1093/ bioinformatics/btq033.

98. Ye J, Zhang Y, Cui H, Liu J, WU Y, Cheng Y, et al. WEGO 2.0: a web tool for analyzing and plotting GO annotations, 2018 update. Nucl Acids Res. 2018; 46(W1):W71-5. https://doi.org/10.1093/nar/gky400.

99. Conesa A, Götz S, García-Gómez JM, Terol J, Talón M, Robles M. Blast2GO: a universal tool for annotation, visualization and analysis in functional genomics research. Bioinformatics. 2005;21(18):3674-6. https://doi.org/10.1 093/bioinformatics/bti610.

100. Supek F, Bošnjak M, Škunca N, Šmuc T. REVIGO summarizes and visualizes long lists of gene ontology terms. PLoS One. 2011;6(7):e21800. https://doi. org/10.1371/journal.pone.0021800.

\section{Publisher's Note}

Springer Nature remains neutral with regard to jurisdictional claims in published maps and institutional affiliations. 